\title{
Layered Architecture for Internet of Things-based Healthcare System: A Systematic Literature Review
}

\author{
Somayeh Nasiri \\ Department of Health Information Management, School of Health Management and Information Sciences, Iran \\ University of Medical Sciences, Tehran, Iran \\ E-mail: nasiri.so@iums.ac.ir, nasiri.him2015@gmail.com
}

Farahnaz Sadoughi (corresponding author)

Department of Health Information Management, School of Health Management and Information Sciences, Iran University of Medical Sciences, Tehran, Iran

E-mail: sadoughi.f@iums.ac.ir

Afsaneh Dehnad

Department of English Language, School of Health Management and Information Sciences, Iran University of Medical Sciences, Tehran, Iran

E-mail: dehnad.a@iums.ac.ir

Mohammad Hesam Tadayon

Iran Telecommunication Research Centre, Tehran, Iran

E-mail: tadayon@itrc.ac.ir

Hossein Ahmadi

Centre for Health Technology, Faculty of Health, University of Plymouth, Plymouth PL4 8AA, United Kingdom E-mail: hossein.ahmadi@plymouth.ac.uk

\section{Overview paper}

Keywords: internet of things, architecture, healthcare system

Received: June 20, 2021

\begin{abstract}
Internet of Things (IoT), known as a new paradigm, has shown to have a significant role in healthcare domains including remote vital sign monitoring systems, physical activity tracking, early disease diagnosis, and prevention of disease risks. Therefore, designing an integrated healthcare system based on Internet of Things is highly dependent on designing a layered architecture pattern. However, there are no comprehensive studies on Internet of Things layered architecture in the healthcare industry. The purpose of this study was to identify and scrutinize different types of layered architecture of Internet of Things in healthcare in terms of functions, and technologies. We evaluated studies proposing layered architecture of Internet of Things based on security aspects (security requirements and solutions). A systematic literature review was conducted by searching IEEE, PubMed, Scopus and Web of Science between 2005 and 2019. We were able to find 47 academic studies based on inclusion and exclusion criteria. We systematically reviewed applied functions and technologies and categorized them into three main layers namely, the perception, network, and application layers. This study also presented a comprehensive classification of sensor types. Only 28 out of 47 studies proposing Internet of Things architecture addressed security aspects among which privacy, authentication, and access control, confidentiality, and integrity had the highest rank. The layered architecture of Internet of Things is needed to provide an integrated framework for healthcare system, make better communication, and enhance the information management process. We suggest several potential solutions for future research directions according to technical, management, and security challenges
\end{abstract}

Povzetek: Podan je pregled literatura za zdravstvene sisteme, ki uporabljajo večnivojske arhitekture interneta stvari.

\section{Introduction}

With the rapid advances in information and communication technologies in recent years, a new paradigm called Internet of Things (IoT) has emerged [1,
2]. IoT is an innovative technology which was first introduced by Kevin Ashton a professor of Massachusetts Institute of Technology (MIT) in 1999 [1, 3]. The term 
"IoT" refers to connecting all physical objects and devices in the real world to the Internet $[4,5]$. In fact, the main concept of emerging IoT is a network of uniquely identifiable and addressable objects (things) which are embedded with sensors, actuators and microprocessors $[6,7]$. These objects can communicate with each other and exchange information based on communication interoperability protocols [8-11]. The term "IoT" is often associated with different names such as "internet of objects", "ambient intelligence", "ubiquitous computing", "pervasive computing", "cyber physical systems" and "machine to machine interaction" [12-17]. According to these statements, IoT technology allows interaction between People to People (P2P), People to Machine (P2M), and Machine to Machine (M2M) [18]. Indeed, the idea behind the emergence of IoT was to emphasize on "the connectivity for anyone and anything at anytime and anyplace" [19]. One of the widely applied definitions is provided by Uckelmann et al. [11], describing IoT as "an integrated set of future internet" which can be described as a dynamic worldwide and ubiquitous network infrastructure of intelligent objects with "self-configuring capabilities". In general, the IoT motto is "having a modern and better life and promoting the life quality". This is possible by connecting a large number of intelligent devices, technologies, and applications [5]. Besides, three main elements including hardware (sensing devices), middleware (tools for storage and analyzing of data), and visualization are the key components which make IoT [20].

Gartner estimated that there would be 25 billion devices connected to the internet by 2020 [9]. These connections facilitate the volume of derived data and supply a wealth of intelligence for management, analysis, planning and decisions-making [18]. The IoT technology is currently used in different applications including smart home, grid, agriculture, transportation, logistics and industrial sectors $[9,21]$. Among them, healthcare sector is considered as one of the most practical and attractive fields for IoT research [22]. Additionally, the IoT has made a significant potential for many medical domains including remote vital signs, patient monitoring systems, fitness programs, and daily physical activity tracking for the elderly and chronic diseases, continuously monitoring people's physiological and mental conditions [19, 23, 24]. In the near future, the way of providing healthcare services will be altered by developing IoT-based healthcare technologies and services like pervasive and ubiquitous healthcare and telemedicine [3].

Presently, traditional healthcare systems merely focus on patient treatment in healthcare facilities; thereby maintaining traditional healthcare systems are often costly and lacking in quality. Furthermore, patients have to be hospitalized during the treatment processes and delivery of healthcare services in hospitals. However, IoT technology will help change the direction of healthcare services delivery from the hospital-centered approach to the person-centered one. With this new approach, patient treatment takes place at home environment by smart phones and wearable technologies such as smart watches and bracelets [2, 3, 25, 26]. Patient-centered approach can promote quality of care and educate patients about selfcare management and enhance the doctor-patient relationship $[2,27]$. This is possible with the help of IoT technology which provides remote patient monitoring by collecting real-time healthcare data and sending critical information to medical staff. It is worth mentioning that many patients need continuous medical monitoring. Additionally, clinicians should have timely access to their patients' medical records in which the information is as an essential and influential resource in improving the healthcare processes and critical decision-making [19, 28]. Hence, IoT supports early disease diagnosis, prevents possible risks, and assists doctors in remote patient monitoring [22, 29].

IoT-based healthcare system is one of the most challenging areas worldwide. The main problem arises from the requirements of IoT technology in which the environment is inherently complex and dynamic and consists of heterogeneous objects [22, 24,30]. Therefore, in order to communicate and share data between different systems, billions of heterogeneous devices should be able to interconnect and interact with each other through the Internet [3,5]. Moreover, with the development of IoT system, novel healthcare services should be integrated into conventional healthcare systems $[25,28,31]$. It is necessary to design a layered architecture which will be able to overcome these new challenges of IoT [32, 33]. Designing the layered architecture pattern is known as an initial and crucial step for implementing IoT technology [34]. The architecture is a backbone for IoT, which helps developers and designers of systems to provide a cohesive principle for implementing this technology to deliver a quality product $[5,35]$. The major goal of designing IoT layered architecture is to provide a common framework for integrating multiple technologies, making better communication, and enhancing information management processes such as sensing, data collecting, transmitting, processing and storage [36-38]. If IoT technologies in each layer are not suitably configured, the IoT system might be exposed to multiple vulnerabilities and problems [30]. Therefore, multi-layered architectural pattern supports different aspects of IoT system deployments such as scalability, modularity, flexibility, configuration, security, and interoperability among heterogeneous systems [5,37,38]. According to this pattern, system components are divided and organized into separated units, called layers [39-41]. This architecture pattern concentrates on grouping relevant functions into distinct layers which present a consistent set of roles and tasks [42].

Although there is evidence of the proposed IoT architectures in the healthcare industry, these architectures are sparse in multiple sources and are only suitable for special application domains, and there is no comprehensive review of IoT layered architecture covering all healthcare domains [6, 43]. Besides, some articles have not investigated IoT layered architecture and they only addressed general detail of IoT structure [44]. There are different architecture styles for IoT including layered, client-server, peer-to-peer, service-oriented, REST and Microkernel architecture. However, many 
articles have overlooked the importance of IoT-based healthcare architecture in a layered way. Layered architecture assists system developers to partition the whole system into layers. Hence, it can provide an indepth examination of the components and functions of each layer. A remarkable capabilities of a layered style is that it can be applied in integration with many other styles, which is not a statement that holds for all styles. Moreover, the advantage of layered architecture is scalability, reusability, and testability. If the layers are inspected in an in depth way, security will be ensured before connecting to healthcare devices and any changes in sensitive areas of the system can be detected [39].

Furthermore, there are no detailed studies demonstrating a well-defined classification of types of sensors, technologies, and functionalities for each layer, and no survey study has separately evaluated architectures in terms of security aspects. Therefore, in an effort to better understand the advancement of IoT technologies and functionalities, a systematic literature review was performed in this area. Thus, the main objective of this study was to present a comprehensive review of related studies regarding the IoT layered architecture in healthcare domains. Accordingly, we formulate four Research Questions (RQS) to be answered on the basis of a comprehensive review as follows:

- RQ1: What are the main application domains of layered architecture for IoT-based healthcare?

- RQ2: What technologies are used in each layer of architecture for IoT-based healthcare?

- RQ3: What main functions are considered in each layer of architecture for IoT-based healthcare?

- RQ4: What are the main aspects of security in the layered architecture for IoT-based healthcare?

The rest of this study is divided into five sections: the research methodology is illustrated in Section 2. The results are presented on the basis of the research aims in Section 3. Finally, discussion and conclusion are explained in Section 4 and 5, respectively.

\section{Research methodology}

This study is a systematic literature review based on a consistent guideline of Preferred Reporting Items for Systematic Reviews and Meta Analyses (PRISMA) provided by Moher et al. [45]. Therefore, we conducted a review of all relevant studies by focusing on well-defined research questions leading to an increase in knowledge and better understanding of the types of layered architecture for IoT-based healthcare.

\subsection{Search strategy}

We conducted a research of four main databases (IEEE, PubMed, Scopus and Web of Science) between 2005 and 2019 to meet the objective of the study. In this way, two major branches of science namely medicine and computer were connected. The above mentioned databases could support a rich source of reliable information regarding the layered architecture for IoT-based healthcare context from different areas of knowledge. Then, we used Boolean operators such as "OR" and "AND" to combine the three groups of terms in searching the studies and constructing the search string as bellow:

("internet of things" OR "internet of objects" OR "ambient intelligence" OR "ubiquitous computing" OR "pervasive computing" OR "heterogeneous sensor" OR "cyber physical system" OR "machine to machine communication") AND (architecture OR framework) AND (e-health OR ehealth OR "healthcare" OR "health care" OR medica* OR health* OR "smart health")

In the next step, all searched studies were imported into EndNote software and duplicate studies were removed. The results are shown in Fig. 1.

\subsection{Inclusion and exclusion criteria}

On the basis of the research objectives, inclusion and exclusion criteria for our systematic literature review were determined (See Table 1).

\begin{tabular}{|c|c|c|}
\hline $\mathbf{I} / \mathbf{E}$ & Criteria & Explanation \\
\hline \multirow{6}{*}{ : } & $\begin{array}{l}\text { Language } \\
\text { type }\end{array}$ & $\begin{array}{l}\text { Studies written in English- } \\
\text { language }\end{array}$ \\
\hline & $\begin{array}{l}\text { Publication } \\
\text { year }\end{array}$ & $\begin{array}{l}\text { Studies published between } 2005 \\
\text { and } 2019\end{array}$ \\
\hline & $\begin{array}{l}\text { Publication } \\
\text { venue }\end{array}$ & $\begin{array}{l}\text { Studies published in peer- } \\
\text { reviewed journals and } \\
\text { international conferences }\end{array}$ \\
\hline & $\begin{array}{l}\text { Research } \\
\text { method }\end{array}$ & $\begin{array}{l}\text { Case studies, experimental } \\
\text { studies, surveys, review articles, } \\
\text { field studies, simulation and } \\
\text { prototyping studies }\end{array}$ \\
\hline & $\begin{array}{l}\text { Research } \\
\text { scope }\end{array}$ & $\begin{array}{l}\text { Studies proposing layered } \\
\text { architecture pattern for IoT } \\
\text { healthcare. }\end{array}$ \\
\hline & $\begin{array}{l}\text { Related } \\
\text { content }\end{array}$ & $\begin{array}{l}\text { Studies describing technologies } \\
\text { and functions used in each layer } \\
\text { of IoT. }\end{array}$ \\
\hline \multirow{4}{*}{.气̃ } & $\begin{array}{l}\text { Without full- } \\
\text { text }\end{array}$ & $\begin{array}{l}\text { The full-text of the studies is } \\
\text { not available. }\end{array}$ \\
\hline & $\begin{array}{l}\text { Non-related } \\
\text { publication } \\
\text { source }\end{array}$ & $\begin{array}{l}\text { Publication source of the } \\
\text { studies is a report, brief report, } \\
\text { book, thesis and dissertation, } \\
\text { editorial letter, commentary, } \\
\text { workshop, poster and } \\
\text { unpublished working study. }\end{array}$ \\
\hline & $\begin{array}{l}\text { Vague } \\
\text { categorization }\end{array}$ & $\begin{array}{l}\text { Studies contain inadequate and } \\
\text { unclear information about the } \\
\text { topic. }\end{array}$ \\
\hline & $\begin{array}{l}\text { Unrelated } \\
\text { contents }\end{array}$ & $\begin{array}{l}\text { Studies reporting non-layered } \\
\text { architecture patterns, describing } \\
\text { technical and semantic issues, } \\
\text { and focusing on IoT } \\
\text { architectures other than } \\
\text { healthcare field (such as } \\
\text { manufactory, agriculture, } \\
\text { transportation, building, } \\
\text { logistics, etc.). }\end{array}$ \\
\hline
\end{tabular}

Table 1: Inclusion criteria and exclusion criteria for selection studies. 


\subsection{Study selection}

The selection process of studies was carried out in two stages. First, the two authors independently reviewed and screened studies based on titles and abstracts, and according to the defined inclusion and exclusion criteria; consequently, the irrelevant studies were removed. In this regard, if a study referred to IoT architecture in healthcare area, it would be screened for the next stage. Second, in order to determine the articles for eligibility, full texts of extracted studies from the previous stage was investigated independently by the authors of this study. Next, according to our research aims, studies were selected if they followed the layered architectural pattern. As a result, a precise step was taken for study selection to reach a consensus. Eventually, we selected 47 studies regarding the layered architecture for IoT-based healthcare system (See Fig. 1).

\subsection{Data extraction and synthesis}

In this step of review, an initial data extraction form was developed to answer the research questions. Relevant items of IoT were extracted from each study in two sections including general information items (country, publication venues, and research type) and specific information items (architecture application domain, applied technologies and functions in each layer, security aspects and findings). The first two authors reviewed the selected studies independently. Any disagreement was resolved by consensus between the two authors and if necessary, the third and fourth author intervened.

\section{Results}

A total of 6706 studies were identified according to our search strategy, where 47 studies $[2,4,7,10,23,25,28$,

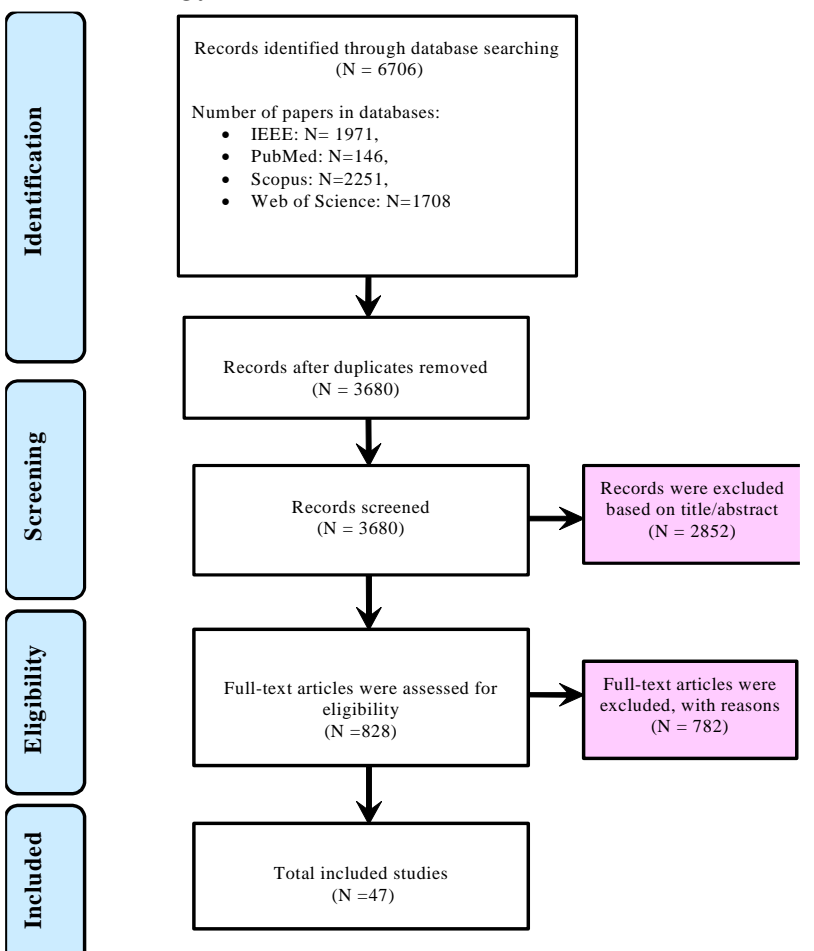

Figure 1: Flow diagram of review process.
31-35, 37, 41, 46-78] according to inclusion and exclusion criteria were included (See Fig. 1). In the following sections, after summarizing and reviewing all studies, we classified the selected studies based on the study characteristics including country, journal and conference names, and research type (Section 3.1). Then, on the basis of the analysis of studies, major findings consisting of application domains of IoT architecture, applied technologies and functions for each layer, and security aspects are presented in Section 3.2.

\subsection{Overview of study characteristics}

\subsubsection{Distribution of studies by country}

As can be seen in Fig. 2, 16 countries have published studies related to IoT layered architecture for various healthcare domains. It is clear from the chart that the majority of studies have been conducted in China $(n=16$; $34.04 \%)$ and India $(n=7 ; 14.89 \%)$. Additionally, the detailed information about other countries is presented in Fig. 2.

\subsubsection{Distribution of studies by the publication venues}

Fig. 3 shows the distribution of 47 selected studies on the layered architecture of IoT-based healthcare, published in 29 journal articles and 18 international conferences.

The five hot venues with the larger number of published studies are shown in Fig. 4. "Applied Mechanics and Materials" ( $\mathrm{n}=4 ; 8.51 \%)$ [47, 50, 54, 69] show the highest rank among the published studies, followed by "Future Generation Computer Systems" [2, 60], " Advanced Materials Research" [49, 75], "Journal of Medical Systems" [71, 72] and "E-Health Networking, Application and Services, HealthCom" [33, 74] $\quad(n=2$ $4.26 \%)$.

\subsubsection{Distribution of studies by research type}

As Fig. 5 shows the distribution of 47 academic studies by research type, 28 of which were of the type of conceptual proposal providing the IoT layered architecture for various healthcare domains without performing any testing and evaluation. Only 11 out of 47 studies validated and experimented the performance of the layered architecture to pre-implementation phase (validation research), and 8 out of 47 studies evaluated the IoT layered architecture to post-implementation phase (evaluation research).

\subsection{Overview of major study findings}

\subsubsection{Distribution of studies by application domains of the IoT layered architecture (RQ1)}

Distribution of studies based on application domains of IoT layered architecture is shown in Table 2. Majority of studies which proposed IoT layered architecture, focused on specific domains of healthcare including diseases $(n=7$, $14.89 \%$ ), ambient assisted living for elderly and disabled 


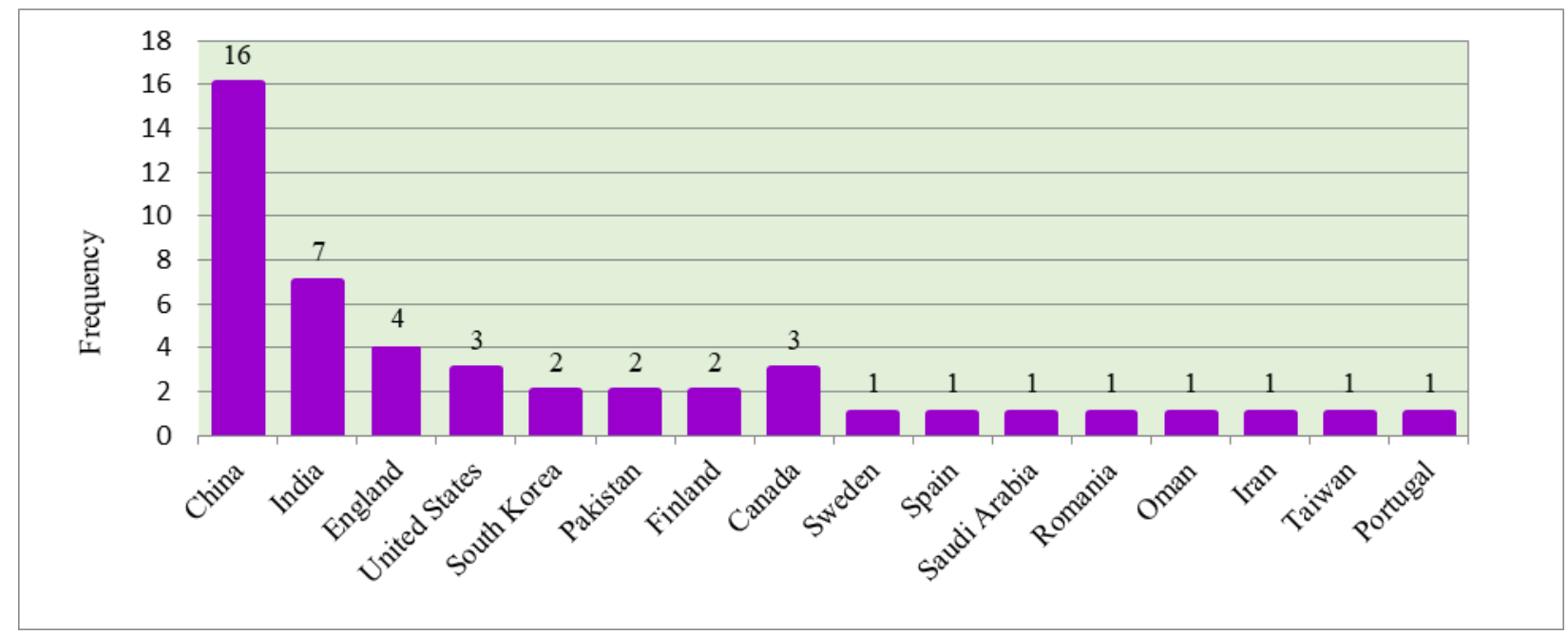

Figure 2: Frequency distribution of selected articles by country.

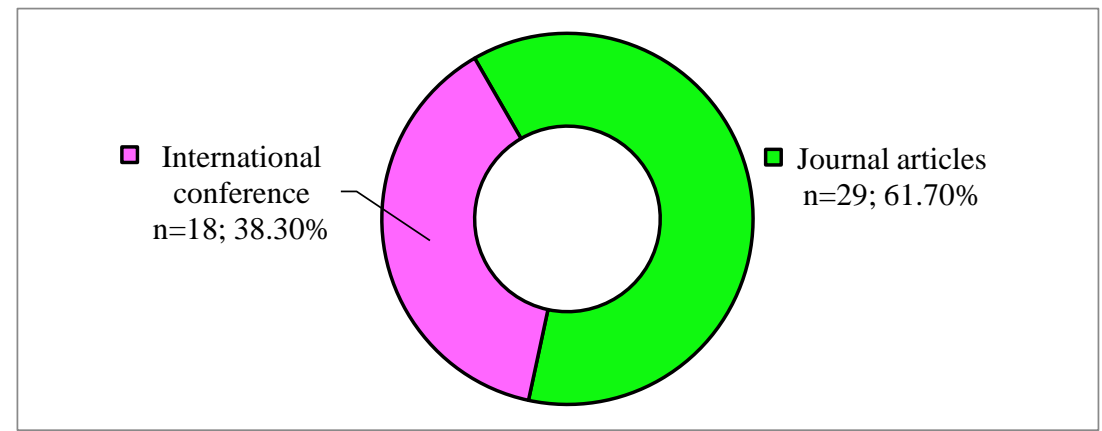

Figure 3: Frequency distribution of selected studies by venue types.

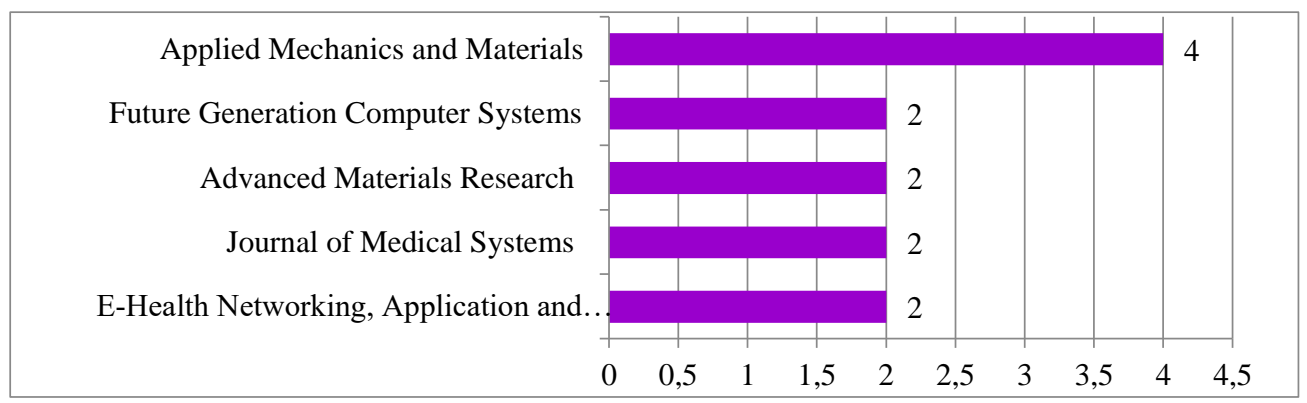

Figure 4: The hot five venues with the highest number of studies.

people $(n=5,10.64 \%)$, smart sports $(n=2,4.26 \%)$ and physical activity $(n=2,4.26 \%)$. Additionally, among the architectures presented, 17 studies $(36.17 \%)$ specifically addressed service management in the healthcare sector including smart hospitals, medical equipment, ICU monitoring, nursing care, smart medication, mental health education, post discharge care management, medical emergency, personalized healthcare services and smart community healthcare services. Moreover, the results showed that 14 out of 47 studies $(29.79 \%)$ proposing IoT layered architecture, belonged to general real-time or remote health monitoring, measuring various parameters including body physiology (e.g., temperature, BP, ECG, EEG, etc.).

\subsubsection{The main technologies and functions in the layered architecture for IoT-based healthcare system (RQ2 and RQ3)}

One of main contributions of this study is that it has systematically identified and categorized IoT-based healthcare technologies and functionalities into a layered architecture. Hence, to answer RQ2 and RQ3, we reviewed 47 studies regarding the IoT architecture in healthcare domains according to functionalities (See Fig. 6) and technologies (Table 3-5 and Fig. 7) and then categorized them into three main layers namely, the perception, network, and application layers. Due to lack of a unified architecture for IoT-based healthcare, and to better understand architectural layers, we followed the architecture proposed by International Telecommunication Union (ITU) [79, 80]. Detailed information on technologies and functionalities identified 


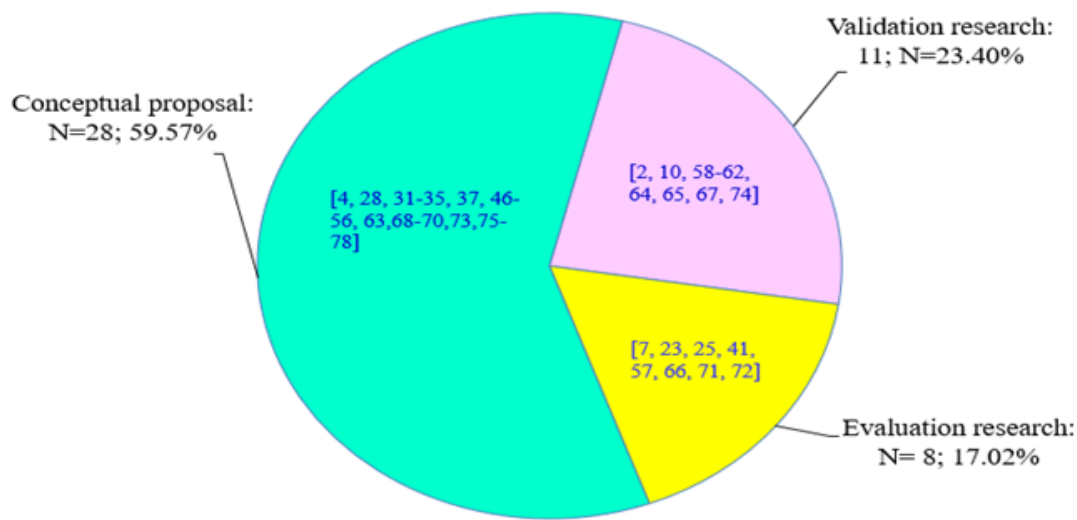

Figure 5: Frequency distribution of selected studies by research type.

in each layer of IoT architecture for healthcare industry are illustrated as follows:

\section{Perception layer}

The first layer of the IoT architecture is called the perception layer which is also known as "sensing" [74], "data collection" [59], "smart objects" [10], "hardware" [41], and "physical layer" [7]. As seen in Fig. 6, a large number of the studies describe the main functions of this layer including object recognition and identification, data sensing and acquisition. This layer consists of physical objects and various types of sensors, mobile devices (e.g., cell phones, tablet PCs, smart phones, PDAs, laptops and pocket PCs), and wearable devices (e.g., smart watches, wristband and fitness band). Depending on the functionality this layer provides, it can be divided into two sub-layers including perception nodes and perception networks [79]. Our findings show that most sensors act as tools for measuring and collecting the data related to the medical status, movement, location and position of a person and environmental conditions. Table 3 shows four categories of the most popular sensors used in IoT-based healthcare system. This category consists of physiological sensors, motion sensors, environmental sensors and position sensors. The following section reports the most important sensors used in the selected studies.

\section{A. Physiological Sensors}

We identified 12 types of physiological sensors used in IoT system as shown in Table 3. In general, these sensors are capable to sense and monitor physical parameters such as body temperature, Electrocardiogram (ECG), Electroencephalogram (EEG), Electromyogram (EMG), Blood Glucose (BG), Blood Pressure (BP), pulse rate and body weight. Some sensors measure emotional parameters and stress level of people. All of these parameters, which are known as vital indicators, continuously measure health condition by wearable devices [81]. According to Table 3, the most common types of physiological sensors belongs to ECG (27 studies), followed by body temperature (22 studies), BP (24 studies), and pulse rate (19 studies); whereas the sensors with the lowest type of frequency is Electrooculography (EOG) (1 study) and weight (3 studies).

\section{B. Motion Sensors}

In Table 3, different types of motion sensors, also called inertial sensors, are shown to have a considerable role in monitoring the motion and activities of human body [82]. Based on our analysis of selected studies, a wide range of

\begin{tabular}{|c|c|c|}
\hline $\begin{array}{l}\text { Application } \\
\text { domains }\end{array}$ & $\begin{array}{l}\text { Number of } \\
\text { studies }\end{array}$ & Reference \\
\hline $\begin{array}{l}\text { Cardiovascular } \\
\text { disease }\end{array}$ & 5 & {$[4,41,49,57,59]$} \\
\hline $\begin{array}{l}\text { Cerebrovascular } \\
\text { disease }\end{array}$ & 1 & {$[67]$} \\
\hline $\begin{array}{l}\text { Diabetes } \\
\text { management }\end{array}$ & 1 & {$[58]$} \\
\hline $\begin{array}{l}\text { Ambient Assisted } \\
\text { living (AAL) }\end{array}$ & 5 & {$[10,32,46,73,74]$} \\
\hline $\begin{array}{l}\text { Smart hospital } \\
\text { management }\end{array}$ & 3 & {$[33,51,61]$} \\
\hline $\begin{array}{l}\text { Medical equipment } \\
\text { management }\end{array}$ & 1 & {$[35]$} \\
\hline ICU monitoring & 1 & {$[71]$} \\
\hline $\begin{array}{l}\text { Medical emergency } \\
\text { management }\end{array}$ & 1 & {$[72]$} \\
\hline $\begin{array}{l}\text { Nursing care } \\
\text { management }\end{array}$ & 3 & {$[28,47,50]$} \\
\hline $\begin{array}{l}\text { Post discharge care } \\
\text { management }\end{array}$ & 1 & {$[31]$} \\
\hline $\begin{array}{l}\text { Smart medication } \\
\text { management }\end{array}$ & 1 & {$[25]$} \\
\hline $\begin{array}{l}\text { Physical activity } \\
\text { monitoring }\end{array}$ & 2 & {$[55,77]$} \\
\hline Smart sports & 2 & {$[23,65]$} \\
\hline $\begin{array}{l}\text { Mental health } \\
\text { education }\end{array}$ & 1 & [54] \\
\hline $\begin{array}{l}\text { Personalized } \\
\text { healthcare services }\end{array}$ & 2 & {$[48,68]$} \\
\hline $\begin{array}{l}\text { Smart community } \\
\text { healthcare services }\end{array}$ & 3 & {$[34,52,75]$} \\
\hline $\begin{array}{l}\text { General real- } \\
\text { time/remote health } \\
\text { monitoring }\end{array}$ & 14 & $\begin{array}{l}{[2,7,37,53,56,60,} \\
62-64,66,69,70, \\
76,78]\end{array}$ \\
\hline
\end{tabular}

Table 2: Application domains of the IoT-based healthcare layered architecture. 
motion sensors including accelerometer $(n=15)$, gyroscope $(n=8)$, magnetometer $(n=6)$, barometric pressure $(n=6)$, piezo vibration $(n=1)$, strain gauge $(n=1)$, impact $(n=1)$ and tilt meter $(n=2)$ were identified. Table 3 shows that accelerometer sensors have the highest rank, while gyroscope, magnetometer, and barometric pressure sensors are ranked second and third among motion sensors.

\section{Environmental Sensors}

According to Table 3, most of environmental sensors are used for monitoring the environmental conditions and changes, such as room temperature, oxygen level and humidity. In addition, some sensors are able to detect and diagnose any smoke, chemical and toxic substances and light. Other environmental sensors are capable of controlling open and close doors or windows, and water leak. As can been observed from Table 3, the lowest and highest types of environmental sensors are related to water leak sensors $(n=1)$ and room temperature $(n=12)$, respectively.

\section{Position sensor}

Positioning systems consist of various sensors which are capable of continuously detecting and tracing position, location, and proximity of a person or an object in real time [83]. According to Table 3, our findings show an extensive range of positioning technologies including GPS $(\mathrm{n}=11)$, ultrasonic devices $(\mathrm{n}=1)$, laser scanners $(n=4)$, and image sensors $(n=12)$ (e.g., camera, video camera, webcam, etc.).

\section{Network layer}

The network layer, known as core layer, is placed in the second layer of IoT architecture. As can be seen in Fig. 6, this layer is responsible for communicating and transmitting the data securely from the lower layer (perception layer) to upper layer (application layer). Network layer contains three sub-layers including access network, core network, and local and wide area network [79]. Fig. 6 depicts the functions of network layer referring to interoperability, pre-processing, data buffering and aggregation. This layer can filter unnecessary data from a great volume of data and ensure a secure communication.

In this section, we present a summary of types of short-range and long-range communication network technologies shown in Table 4 and Table 5. These tables illustrate key properties of technologies according to the type of network, standard, frequency, data rate, range, topology, power consumption and cost) [3, 23, 77, 83-89]. According to Alarifi et al. [83], a group of technologies act as network-based positioning system. Positioning technologies are also employed in network connectivity and communications including RFID, Bluetooth, NearField Communication (NFC), Wi-Fi, Infrared, Ultrawideband (UWB), cellular, ZigBee, Z-wave, Low-power Wireless Personal Area Networks (6LoWPAN) (See Table 4).

\section{Application layer}

The application layer is the topmost layer of the IoT architecture. According to Fig. 6, this layer can be divided into two subsets namely, application support layer and IoT applications.

\section{A. Application support layer}

The Application support layer is responsible for storage, analysis and processing of received data from the lower layer (network layer). This layer is called with different names such as "cloud layer" [28], "processing and storage layer" [66], "service supporting"[67], and "management layer" [65]. As illustrated in Fig. 7, the selected studies show different technologies including cloud computing $(\mathrm{n}=21)$, data mining $(\mathrm{n}=13)$, and Decision Support System (DSS) $(n=16)$. With the help of these technologies, significant and valuable information has been used for a specific purpose such as knowledge discovery, exploratory analysis, and intelligent decision-making (See Fig. 6). According to Fig. 7, only four studies reported fog computing and big data as effective technology for this layer. There were 20 studies discussing data centers as integrated tools for deploying and maintaining all stored data in various sources (e.g., web server, application server, analytic server, database server and storage systems). A data center as a software platform is an indispensable component of IoT architecture to handle and manage received data from gateway networks [10, 90]. Support layer enables management of the entire IoT system such as activities and services. In this respect, 12 studies reported the business process as one of the most important capabilities of the support layer. In IoT-based healthcare systems, the business process can be addressed for determining particular requirements and standards, and defining policies in how data flow is managed, processed, integrated and controlled [31, 74]. Additionally, this layer is the main body of coordination of all activities such as management of the patient medical records (for real-time accessing to history and necessary information), healthcare facilities, equipment and materials and financial issues.

\section{B. IoT Application layer}

IoT application layer is responsible for the delivery of diverse applications and services according to the user's request. According to Fig. 6, basic function of this layer is to display and visualize the information on the central monitoring systems such as nurse station, workstation, touch screen, BeneVision mobile viewer and dashboard. The outcome of this layer will be a visual representation of information in the format of texts, tables, pictures and graphs. Different entities are resided in the application layer as the user (e.g., patient, doctor, nurse, caregiver, administrator, patient's family, technical support team, etc.), location (e.g., hospital, emergency center, clinic, pharmacy supply chain, government agency, home health agency, insurance and other organizations) and technologies [51, 61, 62, 72]. Application layer involves a range of e-health technologies including Electronic Medical Record (EMR) [35, 69], smart e-health monitoring system [7, 53], Tele-consultation [47, 52-54], Tele-surgery [66], e-prescription [25], hospital information system [33, 50, 53, 61], and medication reminder system [56]. Clinicians and medical staff can remotely observe and monitor a patient's vital sign 


\begin{tabular}{|c|c|c|c|}
\hline Category & Sensor type & Function & Reference \\
\hline \multirow{12}{*}{ 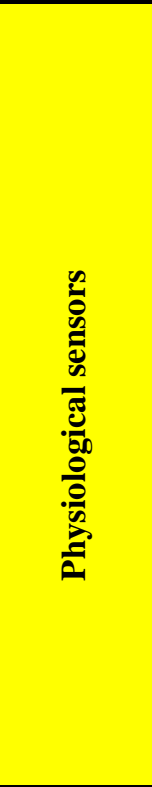 } & ECG & Measuring heart's rhythm and electrical activity & $\begin{array}{l}{[10,23,25,28,34,41,47,49,52,} \\
55-57,59-63,65,66,68-71,73, \\
74,76,77]\end{array}$ \\
\hline & $\mathrm{BP}$ & Measuring blood pressure (systolic and diastolic) & $\begin{array}{l}{[7,34,37,47,49,50,52,53,58,} \\
60-62,64-66,68-74,76,77]\end{array}$ \\
\hline & Body temperature & Measuring body temperature scale of person & $\begin{array}{l}{[7,34,37,47,49,51-53,55,56,} \\
60-62,64-66,68,70-74]\end{array}$ \\
\hline & Pulse rate & Measuring heart beats per minute & $\begin{array}{l}{[7,10,23,28,34,37,47,49,50,} \\
52,53,55,56,58,63,65,72-74]\end{array}$ \\
\hline & $\mathrm{SpO} 2$ & $\begin{array}{l}\text { Monitoring and controlling blood oxygen (oxygen } \\
\text { saturation) level in blood. }\end{array}$ & $\begin{array}{l}{[23,28,34,37,49,52,53,60,61,} \\
63-65,71,73,76]\end{array}$ \\
\hline & Respiratory air flow & $\begin{array}{l}\text { Measuring the activity and function of lung, respiratory } \\
\text { rate and lung volume }\end{array}$ & $\begin{array}{l}{[23,34,37,56,61,65,68,71-74,} \\
76,77]\end{array}$ \\
\hline & BG & Measuring the glucose level in blood sample & {$[53,58,60,62,69,72-74,76]$} \\
\hline & EEG & Measuring electrical signals of the brain & {$[25,60,62,65,68,71,73,76]$} \\
\hline & GSR & $\begin{array}{l}\text { Measuring secretion of sweat gland, and explore } \\
\text { emotional stress and anxiety level }\end{array}$ & {$[23,34,53,60,65,71,77]$} \\
\hline & EMG & \begin{tabular}{|l}
$\begin{array}{l}\text { Measuring electrical activity of muscle during } \\
\text { contractions or at rest }\end{array}$ \\
\end{tabular} & {$[34,63,65,73,76]$} \\
\hline & Weight & Measuring body weight scale of a person & {$[50,58,73]$} \\
\hline & EOG & Measuring eye movements & {$[77]$} \\
\hline \multirow{8}{*}{ 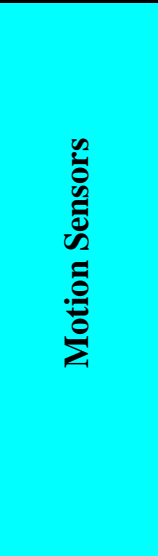 } & Accelerometer & $\begin{array}{l}\text { Measuring linear acceleration of body motion, and } \\
\text { monitoring during walking, standing and sitting }\end{array}$ & $\begin{array}{l}{[10,23,28,37,53,55,56,62,63,} \\
65,68,73,74,76,77]\end{array}$ \\
\hline & Gyroscope & Measuring angular velocity and maintaining orientation & {$[23,55,56,63,65,68,76,77]$} \\
\hline & Magnetometer & $\begin{array}{l}\text { Measuring the strength and direction of the magnetic } \\
\text { field at a particular location such as detecting human } \\
\text { movement direction during watching TV }\end{array}$ & {$[23,52,55,65,68,77]$} \\
\hline & Barometric pressure & $\begin{array}{l}\text { Monitoring human behaviors during climbing up and } \\
\text { down stairs and fall detection }\end{array}$ & {$[10,23,55,63,65,77]$} \\
\hline & Tilt meter & $\begin{array}{|llll|}\begin{array}{l}\text { Monitoring vertical rotation, deflection, and } \\
\text { deformation }\end{array} & & \\
\end{array}$ & {$[65,73]$} \\
\hline & Strain gauge & $\begin{array}{l}\text { Monitoring the motion of the person's body such as } \\
\text { vibration of the vocal cords, movements of joints }\end{array}$ & {$[65]$} \\
\hline & Impact sensor & Detecting the position of the patient such as fall & {$[65]$} \\
\hline & Piezo vibration & Measuring flexibility, vibration, impact and touch & [65] \\
\hline \multirow{8}{*}{ 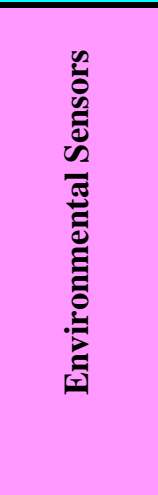 } & Room temperature & Measuring ambient air temperature (indoor/outdoor) & $\begin{array}{l}{[10,23,50,52,53,63,68,71,74,} \\
76-78]\end{array}$ \\
\hline & Hygrometer (humidity) & Measuring ambient moisture (indoor/outdoor) & {$[23,50,63,71,74,77,78]$} \\
\hline & Smoke and toxic substance & $\begin{array}{l}\text { Sensing and detecting the smoke, fire, toxic and } \\
\text { chemical substance and monitors events or malfunctions } \\
\text { lead to raise alarm conditions }\end{array}$ & {$[37,46,50,71,74,76]$} \\
\hline & Noise & Detecting the sound intensity in ambient environment & {$[23,37,52,71]$} \\
\hline & Open/close sensor & Detecting window or door open/close state & {$[65,68,74,77]$} \\
\hline & Light & Detecting the amount of light in the vicinity & {$[23,50,77]$} \\
\hline & Oxygen level & Measuring the amount of Oxygen in the environment & {$[23,71]$} \\
\hline & Leak sensor & Detecting water leak & {$[68]$} \\
\hline \multirow{4}{*}{ : } & Image sensors & \multirow[t]{4}{*}{$\begin{array}{l}\text { Tracking, identifying location, proximity of people or } \\
\text { object in indoor or outdoor environment }\end{array}$} & $\begin{array}{l}{[2,7,10,32,46,47,50,51,53,} \\
54,64,67,68]\end{array}$ \\
\hline & GPS & & $\begin{array}{l}{[10,32,46,47,49,50,56,64,68,} \\
70,77]\end{array}$ \\
\hline & Laser scanners & & {$[32,49,53,63]$} \\
\hline & Ultrasonic & & {$[46]$} \\
\hline \multicolumn{2}{|c|}{ Other sensors and wearable devices } & $\begin{array}{l}\text { e.g. mobile devices, smart cards, wrist band, smart } \\
\text { watch }\end{array}$ & $\begin{array}{l}{[2,4,10,31,46-50,55,56,60,6} \\
67,68,70,75,77,78]\end{array}$ \\
\hline
\end{tabular}

Table 3: The types of sensors used in IoT-based healthcare system by theirs functions. 


\begin{tabular}{|c|c|c|c|c|c|c|c|c|c|c|c|c|c|}
\hline \multirow{2}{*}{\multicolumn{2}{|c|}{ Technology }} & \multicolumn{8}{|c|}{ Properties } & \multicolumn{3}{|c|}{ Function } & \multirow[t]{2}{*}{ Reference } \\
\hline & & 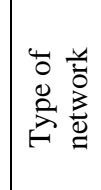 & 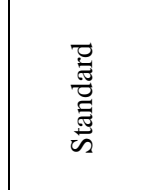 & 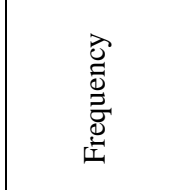 & 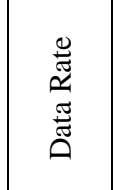 & $\begin{array}{l}\mathscr{D} \\
\tilde{E} \\
\tilde{\Xi}\end{array}$ & $\begin{array}{l}\text { को } \\
\frac{0}{0} \\
\frac{2}{0} \\
0\end{array}$ & 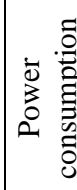 & $\ddot{8}$ & 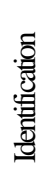 & : & 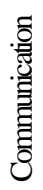 & \\
\hline NFC & & PAN & \begin{tabular}{|l|} 
ISO/IEC \\
18092
\end{tabular} & $\begin{array}{l}13.56 \mathrm{MHz} \\
(\mathrm{ISM})\end{array}$ & $\begin{array}{l}\text { up to } \\
424 \\
\text { kbps }\end{array}$ & $20 \mathrm{~cm}$ & $\mathrm{P} 2 \mathrm{P}$ & Low & Low & $\checkmark$ & $\checkmark$ & $\checkmark$ & $\begin{array}{l}{[23,61,65,68,} \\
77]\end{array}$ \\
\hline RFID & RFID & PAN & $\begin{array}{l}\text { ISO/IEC } \\
15,693\end{array}$ & $\begin{array}{l}125 \mathrm{kHz}-2.45 \\
\mathrm{GHz}\end{array}$ & $\begin{array}{l}40-640 \\
\text { kbps }\end{array}$ & $\begin{array}{l}30-100 \\
\mathrm{~m}\end{array}$ & $\mathrm{P} 2 \mathrm{P}$ & Low & Low & $\checkmark$ & $\checkmark$ & $\checkmark$ & $\begin{array}{l}{[7,23,25,32-} \\
35,37,46,47 \\
49,51,53-55, \\
60,65-68,70, \\
71,74,77]\end{array}$ \\
\hline WSN & & PAN & \begin{tabular}{|l|} 
IEEE \\
802.15 .4
\end{tabular} & $\begin{array}{l}902- \\
928 \mathrm{MHz}\end{array}$ & $\begin{array}{l}20-250 \\
\mathrm{~Kb} / \mathrm{s}\end{array}$ & $\begin{array}{l}20- \\
100 \mathrm{~m}\end{array}$ & $\begin{array}{l}\text { Bus, } \\
\text { Tree, } \\
\text { Star, } \\
\text { Ring, } \\
\text { Mesh } \\
\end{array}$ & High & High & $\checkmark$ & $\checkmark$ & $\checkmark$ & {$[34,63,67]$} \\
\hline Bluetooth & & PAN & \begin{tabular}{|l|} 
IEEE \\
802.15 .1
\end{tabular} & $2.4 \mathrm{GHz}$ & $1 \mathrm{Mb} / \mathrm{s}$ & $<30 \mathrm{~m}$ & Star & $\begin{array}{l}\text { Medi } \\
\text { um }\end{array}$ & Low & - & $\checkmark$ & $\checkmark$ & $\begin{array}{l}{[2,10,23,25,} \\
31,34,37,49, \\
50,56,58,61]\end{array}$ \\
\hline BLE & 1) & PAN & $\begin{array}{l}\text { IEEE } \\
802.15 .4\end{array}$ & $2.4 \mathrm{GHz}$ & $1 \mathrm{Mb} / \mathrm{s}$ & $5-10 \mathrm{~m}$ & Star & $\begin{array}{l}\text { Very } \\
\text { low }\end{array}$ & Low & - & $\checkmark$ & $\checkmark$ & $\begin{array}{l}{[33,41,47,57,} \\
60,73]\end{array}$ \\
\hline IrDA & $\sum$ & PAN & IrDA & $850-900 \mathrm{~nm}$ & $\begin{array}{l}14.4 \\
\text { kbps }\end{array}$ & $0-1 \mathrm{~m}$ & $\mathrm{P} 2 \mathrm{P}$ & Low & Low & - & $\checkmark$ & $\checkmark$ & $\begin{array}{l}{[23,49,52,65,} \\
68,76,77]\end{array}$ \\
\hline UWB & & PAN & \begin{tabular}{|l|} 
IEEE \\
$802.15 .4 a$ \\
and \\
ECMA- \\
368 \\
\end{tabular} & $\begin{array}{l}3.1 \mathrm{G}-10.6 \\
\mathrm{GHz}\end{array}$ & $\begin{array}{l}100- \\
500 \\
\mathrm{Mb} / \mathrm{s}\end{array}$ & $<10 \mathrm{~m}$ & $\mathrm{P} 2 \mathrm{P}$ & Low & High & - & $\checkmark$ & $\checkmark$ & {$[23,51,65]$} \\
\hline ZigBee & & PAN & \begin{tabular}{|l|} 
IEEE \\
802.15 .4
\end{tabular} & $\begin{array}{l}868 / 915 \mathrm{MH} \\
\mathrm{z}, 2.4 \mathrm{GHz}\end{array}$ & $\begin{array}{l}20 \mathrm{k}- \\
250 \\
\mathrm{kbps}\end{array}$ & $\begin{array}{l}10- \\
100 \mathrm{~m}\end{array}$ & $\begin{array}{l}\text { Ad- } \\
\text { hoc, } \\
\text { P2P, } \\
\text { star, } \\
\text { or } \\
\text { mesh }\end{array}$ & $\begin{array}{l}\text { Very } \\
\text { low }\end{array}$ & $\begin{array}{l}\text { Mediu } \\
\mathrm{m}\end{array}$ & - & $\checkmark$ & $\checkmark$ & $\begin{array}{l}{[31-33,35,46,} \\
50-53,55,60, \\
61,67-69,72, \\
73,77]\end{array}$ \\
\hline 6LOWPAN & 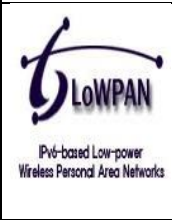 & PAN & \begin{tabular}{|l|} 
IEEE \\
802.15 .4
\end{tabular} & \begin{tabular}{|l}
$868 \mathrm{Mhz}$ \\
(EU) \\
$915 \mathrm{Mhz}$ \\
(USA) \\
$2.4 \mathrm{Ghz}$ \\
(Global)
\end{tabular} & \begin{tabular}{|l|}
$40-250$ \\
$\mathrm{~Kb} / \mathrm{s}$
\end{tabular} & $\begin{array}{l}10-20 \\
\mathrm{~m}\end{array}$ & $\begin{array}{l}\text { Mesh, } \\
\text { star }\end{array}$ & $\begin{array}{l}\text { Very } \\
\text { low }\end{array}$ & Low & - & $\checkmark$ & $\checkmark$ & {$[31,32,65,67]$} \\
\hline Z-Wave & rawa & PAN & $\begin{array}{l}\text { Z-Wave } \\
\text { alliance }\end{array}$ & $900 \mathrm{MHz}$ & $\begin{array}{l}100 \\
\text { Kbps }\end{array}$ & $30 \mathrm{~m}$ & Mesh & $\begin{array}{l}\text { Very } \\
\text { low }\end{array}$ & $\begin{array}{l}\text { Very } \\
\text { low }\end{array}$ & - & $\checkmark$ & $\checkmark$ & [74] \\
\hline Wi-Fi & WiF & LAN & $\begin{array}{l}\text { IEEE } \\
802.11\end{array}$ & $\begin{array}{l}.4 \mathrm{G}-5 \\
\mathrm{GHz}\end{array}$ & \begin{tabular}{|l}
$11-1730$ \\
Mbps
\end{tabular} & $\begin{array}{l}10-100 \\
\mathrm{~m}\end{array}$ & $\begin{array}{l}\text { Star, } \\
\text { mesh }\end{array}$ & Low & High & - & $\checkmark$ & $\checkmark$ & $\begin{array}{l}{[7,28,32,33,} \\
35,46,47,50, \\
51,53,55,57- \\
60,62,65,67- \\
70,72,77,78]\end{array}$ \\
\hline Ethernet & $\frac{6}{6.05}$ & LAN & \begin{tabular}{|l} 
IEEE \\
802.3
\end{tabular} & $100 \mathrm{MHz}$ & \begin{tabular}{|l|}
100 \\
Mbps- \\
10 \\
Gbps
\end{tabular} & $100 \mathrm{~m}$ & $\begin{array}{l}\text { Bus, } \\
\text { star, } \\
\text { P2P }\end{array}$ & Low & Low & - & $\checkmark$ & $\checkmark$ & $\begin{array}{l}{[35,47,51,60,} \\
65,72]\end{array}$ \\
\hline
\end{tabular}

Table 4: Short-range communication technologies for IoT-based healthcare system. 


\begin{tabular}{|c|c|c|c|c|c|c|c|c|c|c|c|c|c|}
\hline \multirow{2}{*}{\multicolumn{2}{|c|}{ Technology }} & \multicolumn{8}{|c|}{ Properties } & \multicolumn{3}{|c|}{ Function } & \multirow[b]{2}{*}{ Reference } \\
\hline & & 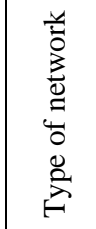 & 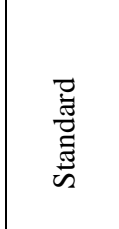 & 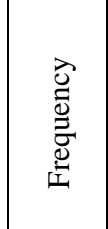 & 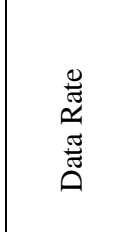 & 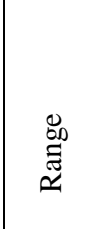 & $\begin{array}{l}\text { के̀ } \\
\frac{0}{0} \\
\stackrel{0}{0} \\
\qquad\end{array}$ & 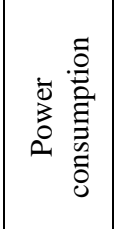 & 范 & 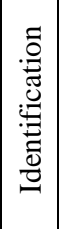 & 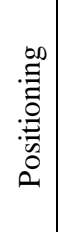 & 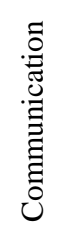 & \\
\hline WiMAX & $\underset{F O R \cup M^{\circ}}{\operatorname{WimAX}}$ & MAN & \begin{tabular}{|l|} 
IEEE \\
802.16
\end{tabular} & $\begin{array}{l}2-66 \\
\mathrm{GHz}\end{array}$ & \begin{tabular}{|l|}
$1 \mathrm{Mb} / \mathrm{s}$ \\
$-1 \mathrm{~Gb} / \mathrm{s}$ \\
(fixed) \\
$50-100$ \\
$\mathrm{Mb} / \mathrm{s}$ \\
(mobile)
\end{tabular} & \begin{tabular}{|l|}
$<50$ \\
$\mathrm{Km}$ \\
\\
\\
\\
\end{tabular} & mesh & Medium & High & - & $\checkmark$ & $\checkmark$ & $\begin{array}{l}{[32,46,67,} \\
68,72,78]\end{array}$ \\
\hline $\begin{array}{c}\text { Mobile } \\
\text { Communication } \\
\text { Network }\end{array}$ & & WAN & \begin{tabular}{|l|} 
2G- \\
GSM, \\
CDMA \\
$2.5-$ \\
GPRS \\
$3 \mathrm{G}-$ \\
UMTS \\
CDMA \\
2000 \\
$4 \mathrm{G}-$ \\
LTE \\
$5 \mathrm{G}$ \\
\end{tabular} & \begin{tabular}{|l|}
450 \\
$\mathrm{MHz}-$ \\
2.6 \\
$\mathrm{GHz}$ \\
\\
\end{tabular} & 1 Gbps & $70 \mathrm{~km}$ & \begin{tabular}{|l|} 
Not \\
available
\end{tabular} & High & Medium & - & $\checkmark$ & $\checkmark$ & 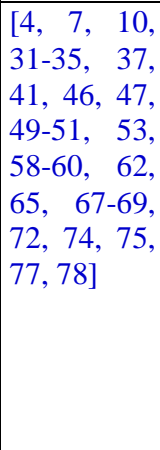 \\
\hline $\begin{array}{c}\text { Satellite } \\
\text { networks }\end{array}$ & syot & WAN & $\begin{array}{l}\text { IEEE } \\
521\end{array}$ & $\begin{array}{l}30-300 \\
\mathrm{GHz}\end{array}$ & $1 \mathrm{Mbps}$ & $\begin{array}{l}6000 \\
\mathrm{~km}\end{array}$ & Star & Low & High & - & $\checkmark$ & $\checkmark$ & {$[53,65,75]$} \\
\hline
\end{tabular}

Table 5: Long-range communication technologies for IoT-based healthcare system.

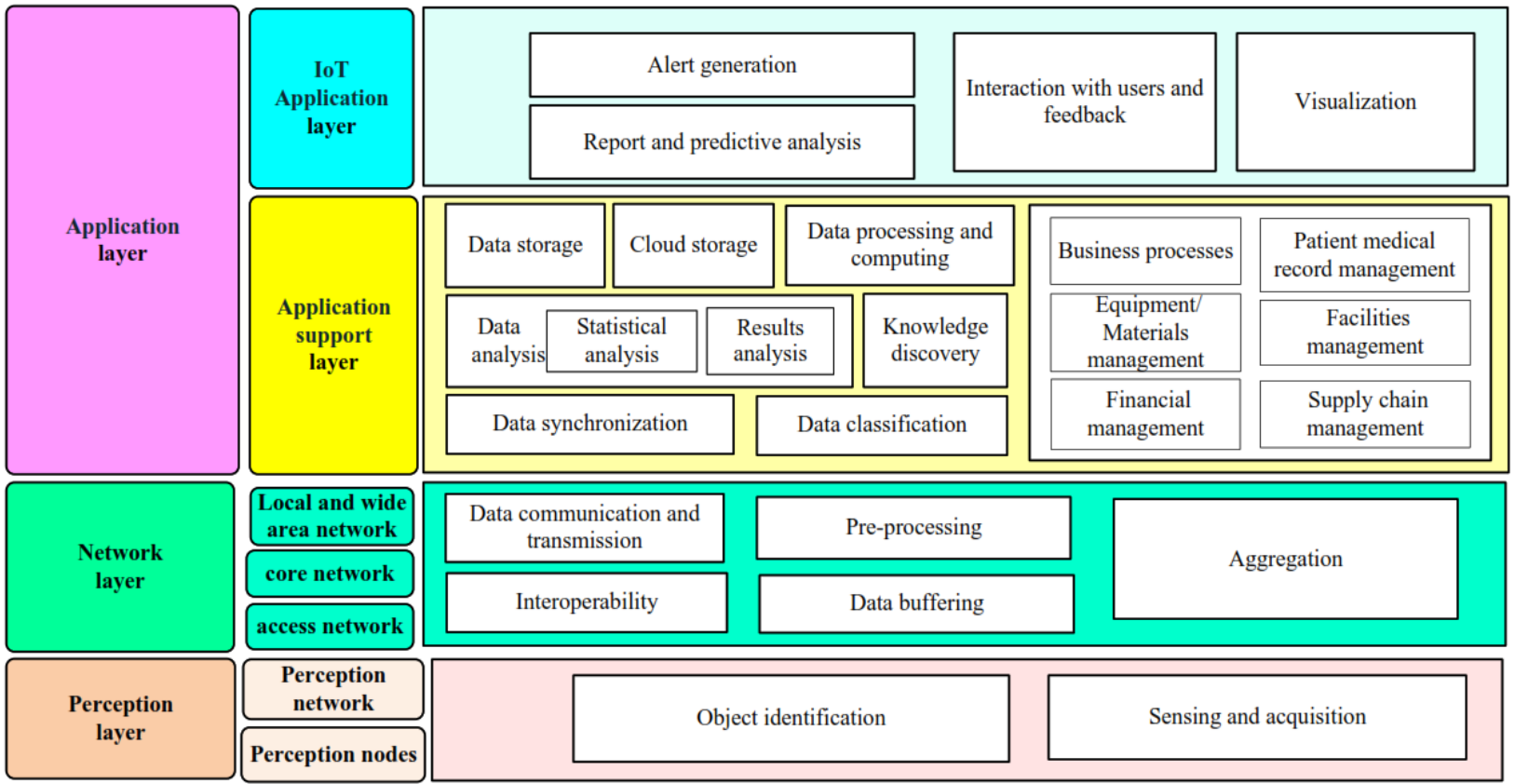

Fig. 6. The main functions used in the layered architecture for IoT-based healthcare system $[2,4,7,10,23,25,28,31$ $35,37,41,46-78]$.

parameters, when the values of the parameters exceed the normal range; alert is automatically sent to medical center and feedback and advisory are provided to the users.
Reporting and prediction are other important functions of this layer described in selected studies. 


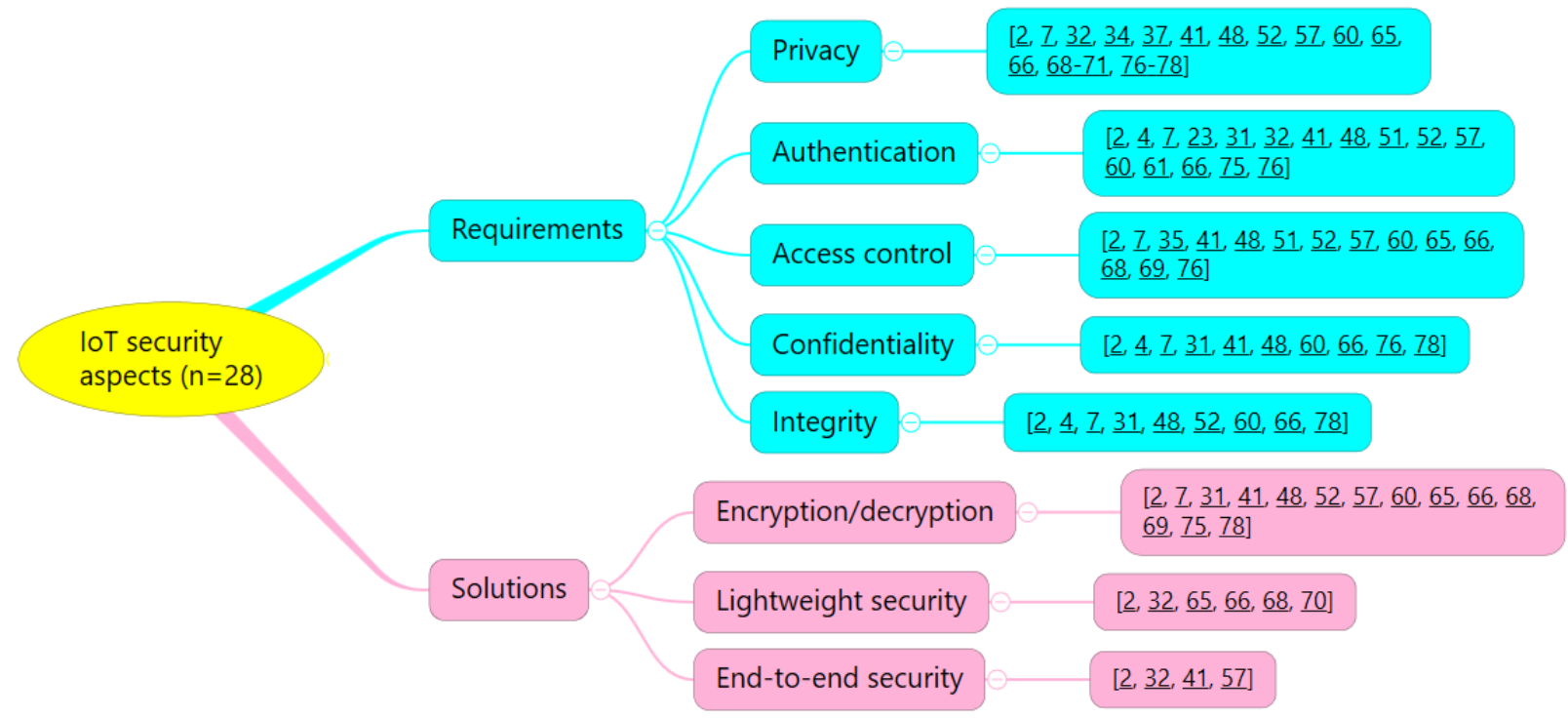

Figure 9: The selected studies about IoT-based healthcare architecture by security requirements and solutions

$[2,4,7,23,31,32,34,35,37,41,48,51,52,57,58,60,61,65,66,68-71,74-78]$.

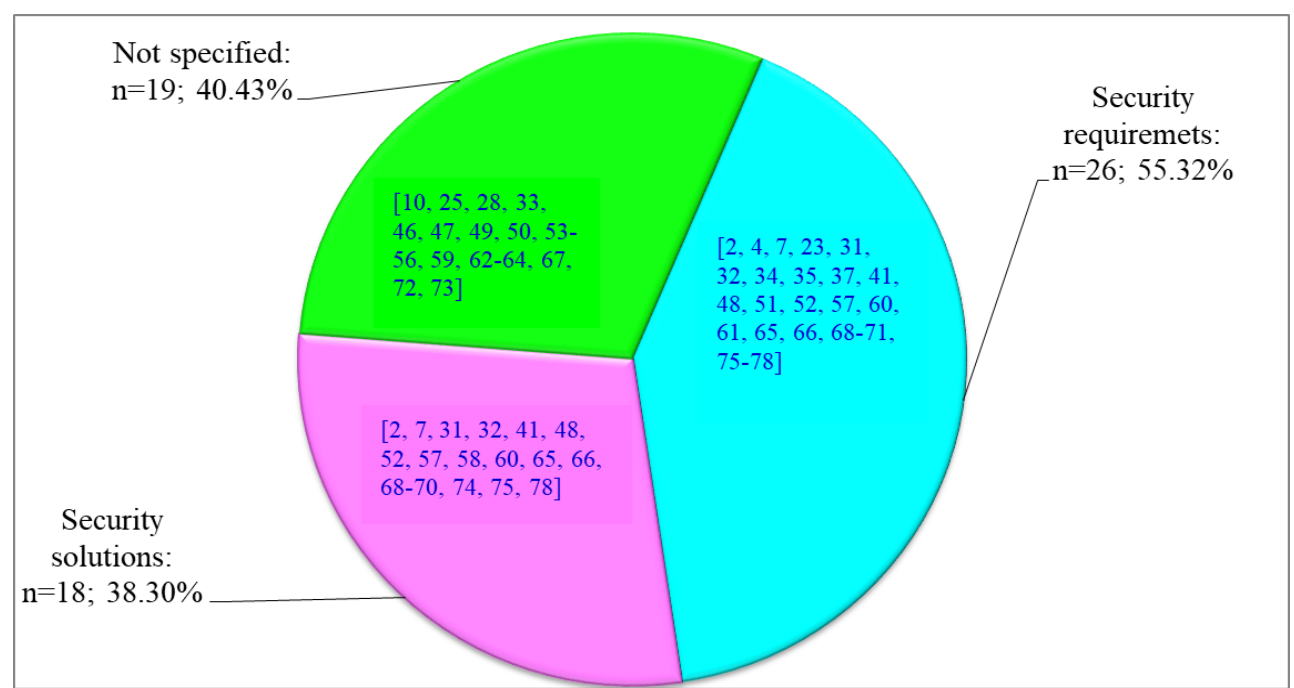

Figure 8: Frequency distribution of selected studies by main security aspects.

\subsubsection{Distribution of studies by main security aspects in IoT architecture (RQ4)}

As shown in Fig. 8, 28 out of 47 studies have included the security aspects of layered IoT architecture. Of these, 26 studies have reported security requirements and 18 studies have reported security solutions. However, 19 of these studies have not addressed any security aspects of IoT architecture. According to Fig. 9, 15 major security requirements in 26 selected studies were identified, among which privacy $(n=19)$, authentication $(n=16)$, access control $(n=14)$, confidentiality $(n=10)$, and integrity $(n=9)$ have the highest rank among other security requirements. Besides, 16 out of 47 studies have focused on security requirements of IoT architecture, as well as providing security solutions including encryption/decryption, lightweight security and end-to-end security.

\section{Discussion}

In our systematic review, 47 academic studies were identified with respect to our formulated research questions between 2012 and 2019. According to our findings, selected studies were from three continents including Asia, Europe and America. It is noteworthy that Asian countries especially China and India have made the most contribution regarding the IoT-based healthcare layered architecture, suggesting an enormous potential and opportunities for research on this topic in Asian countries. Likewise, Talavera et al. [91] showed similar results about IoT layered architecture in agricultural domain. It seems that Asian countries particularly China has the most progress and activities with regard to the projects for IoT layered architecture in different fields.

Based on our analysis of research types, $23.40 \%$ of studies have investigated the validity of the proposed IoT architecture by experiments, simulations, or prototyping. 
Besides, $17.02 \%$ studies evaluated the proposed IoT architecture in the post-implementation phase. Accordingly, one of the strengths of these studies is that they have practically evaluated the accuracy and performance of the architecture or the proposed framework during, before, and after implementation phases of the system. However, over half of the selected studies (28 studies) did not perform any validation or evaluation of the proposed architecture.

\subsection{Application domain (RQ1)}

In terms of application domains of the layered IoT architecture, a large number of published studies $(n=33$; $70.21 \%$ ) have suggested specific application domains of healthcare including diseases, ambient assisted living for the elderly and disabled population, sport and physical activities and management of smart services. Similarly, an architecture has been proposed by Al-Taee et al. [58], mainly focusing on diabetes management. However, few studies have focused on general health monitoring, and measuring different parameters of patient status in real time (e.g., ECG, BG, temperature, BP, respiratory rate, etc.) $[2,7,37,53,56,60,62-64,66,69,70,76,78]$. The results of this study are consistent with the systematic review conducted by Gonzalez et al. [92], remarking that most studies regarding the mHealth systems architecture, focus on diseases, elderly population, disabled people and the athletes.

\subsection{Technologies and functionalities (RQ2 and RQ3)}

Majority of studies did not provide a comprehensive taxonomy of different sensor types. It is important to note that our review study has divided sensor types into four main categories and 31 sub-categories. Based on our analysis, we have identified four groups of fundamental sensors for IoT-based healthcare architecture such as physiological, environmental, motion, and position. Depending on sensor types and their functions, they allow us to perform continuous monitoring of medical parameters, measuring environmental conditions, detecting motion and behavior of people especially the elderly and tracking location, position and proximity of humans or objects $[23,50,52,63,65,68,71,93,94]$.

More importantly, based on our findings, BLE technology has a considerable merit over Bluetooth in terms of low energy consumption for wireless networks. BLE is one of the wireless PAN technologies, known as Bluetooth smart which needs a small battery to be able to run the device for a long time $[6,20]$.

Network layer contains a various types of gateways which are essential to data transmission and are known as intermediate tools for the connectivity between sensors and cloud [18,28,35,93]. According to our results, this layer encompasses well-known short-range and longrange communication technologies which act as a gateway network [65]. The most substantial function of network layer is interoperability which facilitates an effective communication and information exchange across heterogeneous devices $[6,38]$. Based on our analysis of selected studies, it is surprising that most of the studies have proposed IoT architectures without considering the interoperability among devices. Only $25.53 \%$ of the selected studies $(n=12)[2,25,31,32,35,37,60,61,65$, $68,77,78]$ have highlighted the interoperability issue for IoT architecture. Due to an increase in the number of connected objects, interoperability has faced many complexities and barriers such as devices compatibility and identification issues affecting different levels of healthcare systems $[6,18,38]$. In this regard, the need for well-defined communication protocols is a critical and basic component of IoT architecture, allowing devices to interact with each other. It is important to develop universally accepted standards to overcome interoperability problems. In this regard, allocation of a unique Internet Protocol (IP) address such as IPv4 and IPv6 for each device should be taken into consideration. However, IPv4 address alone cannot respond to identify, and protect objects because of scalability and mobility of IoT-based healthcare system with the increasing number of internet connected devices and applications. Consequently, mobile IP for IPv6 address can guarantee billions of large scalable connected devices. This process was facilitated through integrating IPv6 infrastructure and 6LowPAN protocol [22, 32, 38]. On the other hand, Domingo [46] has remarked that the above-mentioned protocols are not suitable in terms of energy efficiency, cost and computation. Therefore, it is necessary to conduct further research on adapting existing protocols and discovering innovative solutions.

Based on our analysis of the selected studies, the third layer has the greatest functionalities and tasks compared with other layers. Overall, the most important task in this layer is processing, computing, storing, analyzing, management and business $[4,31,65,66,69,95]$. One of the most central roles in this layer is the business process. Consequently, it is important that healthcare organizations pay more attention to determining policies and requirements regarding the data management, recourse and service management, equipment management and facilities management $[18,22,31,32,37,61,65,67,93$, 96]. However, many reviewed studies ignored the importance of business activities and provided no solution for business technology algorithms. It is worth mentioning that some proposed architectures for IoT in areas other than healthcare domain have considered the business layer as a separate layer $[5,93]$. However, findings from the two studies of Patel et al. [18] and Darwish et al. [97] are in line with our findings which suggest that business module is an integrated part of service management layer. Therefore, the role of business support systems is essential in the third layer.

In application support layer, cloud computing and big data analytics are known as two novel and ideal technologies [98, 99]. In our review, only $8.51 \%$ of selected studies used these two technologies to overcome the challenges of big data. Given that IoT ecosystem faces massive volume of data generated by medical sensors and numerous devices in real-time, the process and analysis of all the data is necessary $[100,101]$. In healthcare industry, 
cloud computing is a perfect technique to support recourse scalability, flexibility, data storage and computing, and cost saving [100]. Consequently, it is worthwhile to create a smart environment in healthcare industry by emerging cloud computing and IoT. Jalali et al. [34] have remarked that cloud is an imperative and integrated part of an IoTbased healthcare system. Existing cloud computing in IoT architecture facilitates ubiquitous access to resources and services in demand over the network and meets the needs of different medical centers [22]. Guo et al. [102] have suggested that integration of cloud computing and IoT technologies have created a new idea of "cloud of things". It can be inferred that in the near future, cloud computing will have a strong impact on the development of IoT-based information systems. Additionally, big data analytic tools contain different methodologies that can solve data processing and analytics problems in IoT environment and are part of the IoT system requirements $[22,90]$. However, it is surprising that big data technology is used in a small number of proposed architectures.

In application support layer, depending on patient situations and required functions, data processing is done in two forms. Some of the events periodically collect sensory data and perform batch processing at a specific time. In the case of emergency units, it is necessary to immediately collect data via sensor devices, to have realtime processing and giving a fast response to the patient's critical situation [18, 28, 71, 72, 103]. For this reason, IoT medical device manufacturers and developers should take into account both batch and real-time processing to meet users requirements.

Our findings showed that only few studies noted fog computing as the main technology in this layer. Almehmadi et al. [76] discussed that fog computing is one of main IoT technologies expanding cloud computing to new services at the edge of the network. Fog computing supports a variety of services such as latency reduction, real-time systems, mobility, heterogeneity, and interoperability accompanied by the cloud computing. IoT-based healthcare systems should be capable of efficiently functioning to provide continuous vital sign monitoring and real-time medical services without any delay and interruption [2, 76]. Based on our analysis, we believe that it is eminent to consider emerging fog computing in IoT architecture. Azimi et al. [57] evaluated a fog-assisted computing architecture for healthcare IoT systems in terms of response time and latency. These indicators are a critical measure to generate alerts and notifications regarding the status of patients in cases of emergency.

Based on our review, few related studies applied middleware technologies in IoT architecture [33, 50, 54]. Nonetheless, middleware technology, which has an important role in supporting a system, allows overcoming the problems related to IoT-based healthcare systems such as heterogeneity, dependability, interoperability, and decision-making $[6,33,90]$.

The result of our review demonstrated that $27.66 \%$ of the studies have emphasized on data mining playing a crucial role in extracting useful information and knowledge discovery in IoT architecture $[23,31,33,34$,
$63,65,66,68,71,99]$. Nowadays, data mining is used mainly for predicting a range of diseases, assisting with diagnosis and advising physicians in making clinical decisions. But, the potential of data mining is even greater; it concentrates on anomaly-based discoveries to create more informed decisions, and predictive modeling. Overall, application layer is the most important and practical layer which acts as a user interface in terms of providing personalized services to meet the needs of different users including doctors, medical professionals, and patients $[9,28,97,98,104]$. In this layer, the required modules for controlling, monitoring, and producing the alert of the IoT-based healthcare systems should be considered [9]. Because the application layer is in direct association with system users, the authors of this review believe that designing a secure platform for the workstations, smart phones and embedded PCs is essential to the safe protection of the device and the trusted visualization and presentation of data types (texts, images, sounds, reports, etc.).

\subsection{Security aspects (RQ3)}

Considering that IoT-based healthcare devices and applications deal with vital and personal information of patients, they must be protected against any security threats and attacks. Due to the mobility nature of IoT devices connecting objects to global information networks for their access at anytime and anyplace, a wide range of security challenges arise in the healthcare industry. [22]. As a result, IoT security is one of the serious issues in such uncertain and unpredictable environments and can affect the adoption of IoT system. The findings of this review shows that almost half of the studies $[10,25,28,33,46$, $47,49,50,53-56,59,62-64,67,72,73]$ proposing IoT architecture have not provided any comprehensive security solutions or requirements while patient information is sensitive and can be susceptible to hackers during transfer or synchronization stages [105]. Hossain et al. [105] have argued that wearable devices, which are based on IoT, and continuously collect data from patients' ECG, are subject to security breaches. In order to ensure a safe and high quality IoT-based healthcare service, data security and privacy of patients must be protected against any illegal access.

A few of the selected studies have considered IoT security aspects; however, these aspects are only limited to some layers of the IoT architecture $[4,7,32,58,61,67$, $68,71]$. In this regard, Bilal et al. [106] have remarked that all layers of IoT architecture face security challenges and threats. As a result, it is essential that security requirements be considered in all layers of IoT system. For instance, application layer of IoT architecture faces challenges like user privacy and access controls during data sharing, phishing, malware and injection attacks while network layer faces major security problems such as integrity and data confidentially [106]. According to the research performed by Vijayalakshmi et al. [107] the main threats in network layer are eavesdropping, and Denial of Services (DoS)/Distributed Denial of Service (DDoS). 
These threats breach integrity, confidentiality, and availability.

Bilal et al. [106] have suggested that the existing conventional security requirements and standards for IoT architecture is not adequate to protect vulnerable intelligent devices. This finding indicated that a dynamic defense-based mechanism is required for IoT medical devices. Based on our results, it is surprising that some security requirements such as autonomy, safety and fault tolerance are very negligible, whereas according to National Institute of Standards and Technology (NIST) guidelines [108], these requirements are necessary for establishing a resilient system against cyber-attacks. Due to resource constraints of IoT sensors and devices, it is worth mentioning that encryption mechanisms proposed should be lightweight to meet the security requirements [32, 66, 109]. Zhou et al. [110] suggested a secure IoT scheme based on elliptic curves cryptosystem. This scheme is more appropriate and economical for limited resources (e.g. computing complexity, bandwidth, cost, etc.) in IoT environment. It is surprising that only six studies have used lightweight encryption solution for IoT architecture. However, the authors of this study believe an additional work regarding the IoT-based healthcare security should be conducted to further investigate security requirements, vulnerabilities, and mechanisms. IoT in Healthcare industry is a complex area with different users, such as patients, doctors, researchers, insurance payers, and pharmaceutical companies, dealing with large amounts of patient data. Bublitz [78] et al. have offered Blockchain as a distributed and trusted mechanism which can improve issues in the healthcare industry such as security, transparency, data fragmentation, data exchanging, and interoperability. Blockchain can be combined with IoT as an extraordinary solution to enhance patient log control and facilitate secure access to the electronic records. Considering the importance of healthcare information for continuously monitoring patients and the decisions making based on it, we should not ignore the security and quality of the services and information. In this regard, Ebrahimi et al. [111] suggested decentralized trust management model as an efficient mechanism for protecting for IoT based healthcare devices. Alam [112] described IoT with blockchain enables ensure secure data storage and transactions in several industries like e-banking, healthcare monitoring, robotics through a peer-to-peer communication utilizing a shared database without third-party agents.

\subsection{Comparison of related studies}

We reviewed IoT layered architectures from different aspects. In comparison to previous literature on IoT, our study is a strong review in terms of selected keywords, number of databases, various domains of healthcare and extensive research.

Previous studies have only focused on one specific domains of IoT architecture in healthcare industry. For instance, some studies have proposed architectures for nursing care [28], personalized healthcare system [68], disabled people [32], smart sport [65], and physical activities [77] while our study is not exclusive to a specific domain of the disease, population, and service. The present study is a comprehensive review of all IoT architectures in healthcare domains. On the other hand, some studies have not precisely investigated functions and technologies of each layer. Besides, some reviews have used a limited list of terms for search strategies [3, 28, 68]. For example, in a survey study of advanced internet of things for personalized healthcare system, Qi et al. [68] searched only three databases including IEEE Xplore, ACM digital library and ScienceDirect, whereas our review has systematically covered an extensive and complete search of terms related to the IoT and has addressed more databases.

Lopes et al. [32], Meng et al. [69], Mohammed et al. [41] have concentrated on few technologies of IoT architecture, and the review study by Ahmadi et al. [3] have not provided a robust classification of sensors types and functions. This review study has identified and synthesized all the relevant publications on applied technologies in each layer of IoT architecture and presented a comprehensive taxonomy of useful sensors in healthcare industry. More importantly, the present study has scrutinized and evaluated the security aspects in each of the studies proposing layered architecture.

\subsection{Limitations}

In our review study, there are several limitations which can be considered as potential suggestions for future research. First, the data were extracted from the academic journals and international conferences, in which some documents were not closely examined. This includes reports, brief reports, books, theses and dissertations, commentaries, and unpublished studies. Second, the selected studies belong to four main databases (IEEE, PubMed, Scopus, and Web of Science), and other databases (such as ACM, Springer, Willey, etc.) were not searched. Third, we did not perform manual search in the reference lists of the selected studies. Hence, it is possible that some potential information may have been missed. For this reason, further review studies can be conducted to cover other documents. Forth, in our review, studies written in non-English languages were excluded. It is possible that other relevant studies written in different languages might have been missed.

\section{Conclusion and future work}

This study systematically reviewed the current knowledge about different layered architecture of IoT on the basis of the various application domains of healthcare. We were able to identify and summarize types of functions and technologies in each layer and security. The layered architecture perspective of IoT in the healthcare industry covers three main layers: perception layer, network layer, and application layer. In sum, the results of this review are expected to be useful and effective for a large group of communities in the area of the IoT-based healthcare system including academic groups (researchers), healthcare groups (nurses, doctors and medical team 
staff), engineering groups (IT engineers and software developers), and governmental groups (policymakers, managers and decision makers).

As discussed earlier, layered architecture is known as an important and essential step for designing and implementing IoT integrated healthcare system. In fact, multi-layered architectural pattern provides a framework with the aim of integrating various technologies and maintaining interaction of system components with each other to support platforms and application services. Moreover, this architecture facilitates communication and electronic information sharing, and improves remotely healthcare monitoring.

Our research is a landscape for developing more research, providing quality services and real-time access to patient information, designing a suitable platform, and making strategic decisions and investment for IoT deployment. However, we are aware that achieving these goals is not easily reachable, because there are still many challenges for IoT architecture and system deployment. Thus, challenges related to IoT provide directions for further research. The findings of this study provide several recommendations that create opportunities and motivate researchers in future research as follows:

- According to our findings of this review study, there is not a solitary IoT architecture which addresses all issues, including reliability, latency, scalability, and security in the healthcare industry. Therefore, the authors suggest that technical and semantic issues related to IoT-based healthcare system architecture such as scalability, interoperability, standards and communication protocols be considered. Scalable architecture ensures the proper functioning of IoT networks and supports the connectivity of increasing number of devices and sensor nodes. In the healthcare sector, various applications, devices and protocols are delivered by different vendors. Hence, interoperable architecture approaches are recognized as solutions to overcome the heterogeneous networks allowing data sharing throughout IoT system. More importantly, security and privacy issues are the significant problems in IoT architecture. The security issue is related to all layers of IoT architecture which are exposed to different types of attacks and threats, which are areas for investigating in healthcare industry.

- The IoT architecture deals with diversity of high and low cost technologies which also have constraints in terms of power consumption and data storage. Thus, designing a model for cost analysis of IoT devices and choosing an economical solution in terms of energy management is essential.

- The findings of this study indicate that business processes, data flow management and policy determination are main functions which should be considered for IoT-based healthcare architecture. As a result, designing a comprehensive business model for IoT-based healthcare can be considered as a valuable work.
- $\quad$ Given that IoT has faced a massive volume of data which are generated from heterogonous IoT devices, emerging technologies such as cloud computing and big data with IoT would provide a significant opportunity to expand more research in healthcare systems.

- In order to achieve the successful implementation and widespread adoption of IoT system in healthcare industry, it is suggested that proposed IoT architecture be tested and evaluated before implementing the actual system.

\section{Conflicts of Interest}

The authors declare that there is no conflict of interests regarding the publication of this article.

\section{Acknowledgements}

This study has been funded and supported by Iran University of Medical Sciences (Grant Number: 94-04136-26859).

\section{References}

[1] Avachat V, Gupta P. A study of Semantic Middleware for Internet of Things. International Journal of Advanced Research in Computer Science and Software Engineering. 2014; 4(12): 23-7.

[2] Moosavi SR, Gia TN, Nigussie E, Rahmani AM, Virtanen S, Tenhunen H, et al. End-to-end security scheme for mobility enabled healthcare Internet of Things Future Generation Computer Systems 2016; 64: 108-24

https://doi.org/10.1016/j.future.2016.02.020

[3] Ahmadi H, Arji G, Shahmoradi L, Safdari R, Nilashi M, Alizadeh M. The application of internet of things in healthcare: a systematic literature review and classification. Universal Access in the Information Society 2019; 18(4): 837-69. https://doi.org/10.1007/s10209-018-0618-4

[4] Ungurean I, Brezulianu A. An Internet of Things Framework for Remote Monitoring of the HealthCare Parameters. Advances in Electrical and Computer Engineering 2017; 17(2): 11-6. https://doi.org/10.4316/aece.2017.02002

[5] Al-Fuqaha A, Guizani M. Mohammadi M, Aledhari M, Ayyash M. Internet of Things: A Survey on Enabling Technologies, Protocols, and Applications, IEEE Communications Surveys \& Tutorials 2015; 17(4): 2347-76. https://doi.org/10.1109/comst.2015.2444095

[6] Sethi P, Sarangi SR. Internet of things: architectures, protocols, and applications. Journal of Electrical and Computer Engineering. 2017; 2017(1):1-25. https://doi.org/10.1155/2017/9324035

[7] Sharma D, Jinwala D. Functional encryption in IoT E-Health care system. Lecture Notes in Computer Science (including subseries Lecture Notes in 
Artificial Intelligence and Lecture Notes in Bioinformatics), 2015. p. 345-63. https://doi.org/10.1007/978-3-319-26961-0_21

[8] Pticek M, Podobnik V, Jezic G, Beyond the internet of things: The social networking of machines, International Journal of Distributed Sensor Networks 2016; 12(6): 1-15. https://doi.org/10.1155/2016/8178417

[9] Ray PP. A survey on internet of things architectures. Journal of King Saud University Computer and Information Sciences 2016; 30(3): 291-319. https://doi.org/10.1016/j.jksuci.2016.10.003

[10] Yang L, Ge Y, Li W, Rao W, Shen W, A home mobile healthcare system for wheelchair users, In: Proceeding of the 2014 IEEE 18th International Conference on Computer Supported Cooperative Work in Design (CSCWD), 2014, pp. 609-14 https://doi.org/10.1109/cscwd.2014.6846914

[11] Uckelmann D, Harrison M, Michahelles F. An Architectural Approach Towards the Future Internet of Things. Berlin, Heidelberg: Springer Berlin Heidelberg; 2011, pp. 1-24. https://doi.org/10.1007/978-3-642-19157-2_1

[12] Katole B, Sivapala M, Suresh V. Principle elements and framework of internet of things. International Journal Of Engineering And Science 2013; 3(5): 24-9. https://doi.org/10.4028/ www.scientific.net/ amm.303-306.2144

[13] Konstantinidis EI, Bamparopoulos G, Billis A, Bamidis PD. Internet of things for an age-friendly healthcare, Studies in health technology and informatics 2015; 210: 587-91. https://doi.org/ 10.3233/978-1-61499-512-8-587

[14] Madakam S, Ramaswamy R, Tripathi S. Internet of Things (IoT): A literature review. Journal of Computer and Communications 2015; 3(05): 16473. https://doi.org/ 10.4236/jcc.2015.35021

[15] Pang Z, Chen Q, Han W, Zheng L. Value-centric design of the internet-of-things solution for food supply chain: Value creation, sensor portfolio and information fusion. Information Systems Frontiers. 2015; 17(2): 289-319.

https://doi.org/10.2991/emim-15.2015.61

[16] Aeman N, Patil DVM. Internet of things is an advanced concept of ICT for better human life. International journal of pure and applied research in engineering and technology 2016; 4(9): 115863.

[17] Ray PP. Towards an Internet of Things based architectural framework for defence. In: Proceeding of the 2015 International Conference on Control, Instrumentation, Communication and Computational Technologies (ICCICCT), 2015, pp. 411-6.

https://doi.org/10.1109/ICCICCT.2015.7475314

[18] Patel KK, Patel SM, Professor PSA. Internet of Things-IOT: definition, characteristics, architecture, enabling technologies, application \& future challenges. Int J Eng Sci Comput 2016; 6(5): 6122-31. https://doi.org/ 10.4010/2016.1482
[19] Chiuchisan I, Costin HN, Geman O. Adopting the Internet of Things technologies in health care systems. In: Proceeding of the 2014 International Conference and Exposition on Electrical and Power Engineering (EPE), 2014, pp. 532-5. https://doi.org/10.1109/ICEPE.2014.6969965

[20] Hegde SG, Naveen Soumyalatha. Internet of things (IoT): A study on architectural elements, communication technologies and applications. International Journal of Advanced Research in Computer and Communication Engineering 2016; 5(9): 189-93. https://doi.org/10.17148/IJARCCE.2016.5943

[21] Ara T, Shah PG, Prabhakar M. Internet of Things Architecture and Applications: A Survey. Indian Journal of Science and Technology 2016; 9(45): 17. https://doi.org/10.17485/ijst/2016/v9i45/106507

[22] Islam SMR, Kwak D, Kabir MH, Hossain M, Kwak KS, The internet of things for health care: A comprehensive survey, IEEE Access 2015; 3: 678708 . https://doi.org/10.1109/ACCESS.2015.2437951

[23] Bhatia M, Sood SK. A comprehensive health assessment framework to facilitate IoT-assisted smart workouts: A predictive healthcare perspective. Computers in Industry 2017; 92-93: 50-66.

https://doi.org/10.1016/j.compind.2017.06.009

[24] Kolenik T, Gams M. Persuasive technology for mental health: One step closer to (mental health care) equality? IEEE Technology and Society Magazine. 2021;40(1):80-6.

https://doi.org/10.1109/MTS.2021.3056288

[25] Yang G, Xie L, Mäntysalo M, Zhou X, Pang Z, Xu LD, et al. A Health-IoT Platform Based on the Integration of Intelligent Packaging, Unobtrusive Bio-Sensor, and Intelligent Medicine Box. IEEE Transactions on Industrial Informatics 2014; 10(4): 2180-91. https://doi.org/10.1109/TII.2014.2307795

[26] Qiu T, Chen N, Li K, Atiquzzaman M, Zhao W, How Can Heterogeneous Internet of Things Build our Future: A Survey. IEEE Communications Surveys \& Tutorials 2018: 20(3) 1-18. https://doi.org/10.1109/COMST.2018.2803740

[27] Ahmadi M, Jeddi FR, Gohari MR, Sadoughi F, A review of the personal health records in selected countries and Iran, Journal of medical systems 2012; 36(2): 371-82. https://doi.org/10.1007/s10916-010-9482-3

[28] Mieronkoski R, Azimi I, Rahmani AM. Aantaa R, Terava V, Liljeberg P, et al. The Internet of Things for basic nursing care-A scoping review, International journal of nursing studies $2017 ; 69$ 78-90. https://doi.org/10.1016/j.ijnurstu.2017.01.009

[29] Darshan KR, Anandakumar KR. A comprehensive review on usage of Internet of Things (IoT) in healthcare system. In: Proceeding of the 2015 International Conference on Emerging Research in Electronics, Computer Science and Technology (ICERECT), 2015, pp. 132-6. https://doi.org/10.1109/erect.2015.7499001 
[30] Rahman AFA, Daud M, Mohamad MZ. Securing sensor to cloud ecosystem using internet of things (IoT) security framework. In: Proceeding of the International Conference on Internet of things and Cloud Computing, Cambridge, United Kingdom, 2016, pp. 1-5.

https://doi.org/10.1145/2896387.2906198

[31] Vargheese R, Viniotis Y. Influencing data availability in IoT enabled cloud based e-health in a 30 day readmission context, In: Proceeding of the 10th IEEE International Conference on Collaborative Computing: Networking, Applications and Worksharing, 2014, pp. 475-80. https://doi.org/10.4108/icst.collaboratecom.2014.2 57621

[32] Lopes NV, Pinto F, Furtado P, Silva J. IoT architecture proposal for disabled people. In: Proceeding of the International Conference on Wireless and Mobile Computing, Networking and Communications, 2014, pp. 152-8. https://doi.org/ 10.1109/WiMOB.2014.6962164

[33] Pir A, Akram MU, Khan MA. Internet of things based context awareness architectural framework for HMIS. In: Proceeding of the 2015 17th International Conference on E-Health Networking, Application and Services, HealthCom 2015, 2015, pp. 55-60.

https://doi.org/ 10.1109/HealthCom.2015.7454473

[34] Jalali R, El-Khatib K, McGregor C, Smart city architecture for community level services through the internet of things, In: Proceeding of the 2015 18th International Conference on Intelligence in Next Generation Networks, ICIN 2015, 2015, pp. 108-13. https://doi.org/10.1109/ICIN.2015.7073815

[35] Mi YW, Wu RC, Li YY, Zhang K, Wang W, Li T. Architecture and the key technologies of medical equipment management system based on the internet of things. J Comput Theor Nanosci 2016; 13(12): 9722-7.

https://doi.org/10.1166/jctn.2016.5914

[36] Muhammad AP, Akram MU, Khan MA. Survey based analysis of internet of things based architectural framework for hospital management system. In: Proceeding of the 2015 13th International Conference on Frontiers of Information Technology (FIT), 2015, pp. 271-6. https://doi.org/10.1109/FIT.2015.54

[37] Gupta N, Saeed H, Jha S, Chahande M. Pandey S, Implementation of an IOT framework for smart healthcare. In: Proceeding of the 2017 International conference of Electronics, Communication and Aerospace Technology (ICECA), 2017, pp. 622-7. https://doi.org/10.1109/ICECA.2017.8203613

[38] Tokognon CJA, Gao B, Tian GY, Yan Y. Structural Health Monitoring Framework Based on Internet of Things: A Survey. IEEE Internet of Things Journal 2017; 4(3): 619-35. https://doi.org/10.1109/jiot.2017.2664072

[39] Croes E, Hoepman J-H. Software architectural styles in the internet of things. Netherlands: Radboud University Nijmegen; 2015.
[40] Sancho G, Tazi S, Villemur T. A semantic-driven auto-adaptive architecture for collaborative ubiquitous systems. In: Proceeding of the Proceedings of the 5th international conference on Soft computing as transdisciplinary science and technology, 2008, pp. 650-5.

https://doi.org/10.1145/1456223.1456354

[41] Mohammed J, Lung CH, Ocneanu A, Thakral A, Jones C, Adler A. Internet of Things: Remote Patient Monitoring Using Web Services and Cloud Computing. In: Proceeding of the 2014 IEEE International Conference on Internet of Things (iThings), and IEEE Green Computing and Communications (GreenCom) and IEEE Cyber, Physical and Social Computing (CPSCom), 2014, pp. 256-63. https://doi.org/10.1109/iThings.2014.45

[42] Meier JD, Hill D. Microsoft application architecture guide, 2nd Edition. United States: Microsoft Corporation; 2009. p. 560.

[43] Abdmeziem MR, Tandjaoui D, Romdhani I. Architecting the Internet of Things: State of the Art. ed. Springer International Publishing, Cham, 2016.

https://doi.org/10.1007/978-3-319-22168-7_3

[44] Mustafa T, Varol A. Review of the internet of things for healthcare monitoring. 2020 8th International Symposium on Digital Forensics and Security (ISDFS); 1-2 June 2020. https://doi.org/ 10.1109/ISDFS49300.2020.9116305

[45] Moher D, Liberati A, Tetzlaff J, Altman DG. Preferred reporting items for systematic reviews and meta-analyses: the PRISMA statement. BMJ 2009; 339: b2535.

https://doi.org/10.1136/bmj.b2535

[46] Domingo MC. An overview of the Internet of Things for people with disabilities. Journal of Network and Computer Applications 2012; 35(2): 584-96. doi:

https://doi.org/10.1016/j.jnca.2011.10.015

[47] Huang JM. Research on Application of Internet of Things in Nursing Home. Applied Mechanics and Materials. 2013;303-306:2153-6.

https://doi.org/10.4028/www.scientific.net/amm.3 03-306.2153

[48] Lee JD, Yoon TS, Chung SH, Cha HS. ServiceOriented Security Framework for Remote Medical Services in the Internet of Things Environment. Healthcare Informatics Research 2015; 21(4): 271 82. https://doi.org/10.4258/hir.2015.21.4.271

[49] Lu DX, Liu T. The Developing of Medical System in Information Age. dvanced Materials Research. 2013;749: 139-43. https://doi.org/10.4028/ www.scientific.net/amr.749.139

[50] Wang D, Ge WC, Sun LP, Li JH. Neonatal Nursing Information System Based on Internet of Things. Applied Mechanics and Materials. 2013; 401403:1927-30. https://doi.org/10.4028/ www.scientific.net/amm.401-403.1927

[51] Yu L, Lu Y, Zhu X. Smart hospital based on internet of things. Journal of Networks 2012; 7(10): 1654-61. 
https://doi.org/10.4304/jnw.7.10.1654-1661

[52] Wu Z, Liu LH, Huang YS, Wu XM. A community health service architecture based on the internet of things on health-care. In: Proceeding of the IFMBE Proceedings, 2013, pp. 1317-20. https://doi.org/10.1007/978-3-642-29305-4_345

[53] Wang X. The architecture design of the wearable health monitoring system based on internet of things technology. Int J Grid Util Comput 2015; 6(3-4): 207-12.

https://doi.org/10.1504/IJGUC.2015.070681

[54] Wang Y. The Construction of the Psychological Health Education Platform Based on Internet of Things. Applied Mechanics and Materials. 2014; 556-562:6711-5.

https://doi.org/10.4028/www.scientific.net/amm.5 56-562.6711

[55] Qi J, Yang P, Fan D, Deng Z. A survey of physical activity monitoring and assessment using internet of things technology. In: Proceeding of the Proceedings - 15th IEEE International Conference on Computer and Information Technology, CIT 2015, 14th IEEE International Conference on Ubiquitous Computing and Communications, IUCC 2015, 13th IEEE International Conference on Dependable, Autonomic and Secure Computing, DASC 2015 and 13th IEEE International Conference on Pervasive Intelligence and Computing, PICom 2015, 2015, pp. 2353-8. https://doi.org/10.1109/CIT/IUCC/DASC/PICOM .2015 .348

[56] Keh HC, Shih CC, Chou KY, Cheng YC, Ho HK, $\mathrm{Yu} \mathrm{PY}$, et al. Integrating unified communications and internet of $\mathrm{M}$-health things with micro wireless physiological sensors. Journal of Applied Science and Engineering 2014; 17(3): 319-28. https://doi.org/10.6180/jase.2014.17.3.12

[57] Azimi I, Anzanpour A, Rahmani AM, Pahikkala T, Levorato M, Liljeberg P, et al. HiCH: Hierarchical Fog-Assisted Computing Architecture for Healthcare IoT. ACM Trans Embed Comput Syst 2017; 16(5s): 1-20.

https://doi.org/10.1145/3126501

[58] Al-Taee MA, Al-Nuaimy W, Al-Ataby A, Muhsin $\mathrm{ZJ}$, Abood SN. Mobile health platform for diabetes management based on the Internet-of-Things. In: Proceeding of the 2015 IEEE Jordan Conference on Applied Electrical Engineering and Computing Technologies (AEECT), 2015, pp. 1-5. https://doi.org/10.1109/AEECT.2015.7360551

[59] Li J, Zhou H, Zuo D, Hou KM, De Vaulx C. Ubiquitous health monitoring and real-time cardiac arrhythmias detection: a case study. Bio-medical materials and engineering 2014; 24(1): 1027-33. https://doi.org/10.3233/bme-130900

[60] Farahani B, Firouzi F, Chang V, Badaroglu M, Constant N, Mankodiya K. Towards fog-driven IoT eHealth: Promises and challenges of IoT in medicine and healthcare. Future Generation Computer Systems 2018; 78: 659-76. https://doi.org/10.1016/j.future.2017.04.036
[61] Thangaraj M, Ponmalar PP, Anuradha S. Internet Of Things (IOT) Enabled Smart Autonomous Hospital Management System - A Real World Health Care Use Case with the Technology Drivers. In: Proceeding of the 2015 IEEE International Conference on Computational Intelligence and Computing Research (ICCIC), 2015. pp. 174-81. https://doi.org/10.1109/ICCIC.2015.7435678

[62] Khan SF. Health Care Monitoring System in Internet of Things (IoT) by Using RFID. In: Proceeding of the IEEE's 6th International Conference on Industrial Technology and Management (ICITM 2017), 2017. pp. 198-204. https://doi.org/ 10.1109/ICITM.2017.7917920

[63] Ullah K, Shah MA, Zhang SJ. Effective Ways to Use Internet of Things in the Field of Medical and Smart Health Care. 2016. pp. 372-9. https://doi.org/10.1109/intelse.2016.7475151

[64] Jingjing Y, Shangfu H, Xiao Z, Benzhen G, Yu L, Beibei D, et al. Family health monitoring system based on the four sessions internet of things. Telkomnika Telecomun Compt Electr Control 2015; 13(1): 314-20. https://doi.org/10.12928/ TELKOMNIKA.v13i1.1265

[65] Ray PP. Generic Internet of Things architecture for smart sports. In: Proceeding of the 2015 International Conference on Control Instrumentation Communication and Computational Technologies, ICCICCT 2015, 2015, pp. 405-10. https://doi.org/10.1109/ICCICCT.2015.7475313

[66] Kocabas O, Soyata T, Aktas MK. Emerging Security Mechanisms for Medical Cyber Physical Systems. IEEE/ACM Trans Comput BioL Bioinf 2016; 13(3): 401-16. https://doi.org/10.1109/TCBB.2016.2520933

[67] Wang X, Bie R, Sun Y, Wu Z, Zhou M, Cao R, et al. The architecture of an automatic eHealth platform with mobile client for cerebrovascular disease detection. J Med Internet Res 2013; 15(8). https://doi.org/10.2196/mhealth.2550

[68] Qi J, Yang P, Min G, Amft O, Dong F, Xu L. Advanced internet of things for personalised healthcare systems: A survey. Pervasive and Mobile Computing 2017; 41: 132-49.

https://doi.org/10.1016/j.pmcj.2017.06.018

[69] Meng XJ, Cui HQ, Hua R. An IoT-Based Remote Health Monitoring and Management System. In: Proceeding of the Applied Mechanics and Materials, 2014, pp. 1176-9.

https://doi.org/ 10.4018/978-1-7998-2584-5.ch014

[70] Wan J, Al-Awlaqi M, Li MS, O'Grady M, Gu X, Wang $J$, et al. Wearable IoT enabled real-time health monitoring system. Eurasip J Wireless Commun Networking 2018. https://doi.org/10.1186/s13638-018-1308-x.

[71] Bhatia M, Sood SK. Temporal Informative Analysis in Smart-ICU Monitoring: M-HealthCare Perspective. J Med Syst 2016; 40(8): 190. https://doi.org/10.1007/s10916-016-0547-9 
[72] Rathore MM, Ahmad A, Paul A, Wan J, Zhang D. Real-time Medical Emergency Response System: Exploiting IoT and Big Data for Public Health. J Med Syst 2016; 40(12). https://doi.org/10.1007/s10916-016-0647-6

[73] Ray PP. Home Health Hub Internet of Things (H(3)IoT): An Architectural Framework for Monitoring Health of Elderly People. In: Proceeding of the 2014 International Conference on Science Engineering and Management Research (ICSEMR), 2014, pp. 1-3. https://doi.org/10.1109/ICSEMR.2014.7043542

[74] Ngo Manh K, Saguna S, Mitra K, Ahlund C. IReHMo: An efficient IoT-based remote health monitoring system for smart regions, In: Proceeding of the 2015 17th International Conference on E-Health Networking, Application and Services, HealthCom 2015, 2015, pp. 563-8. https://doi.org/10.1109/HealthCom.2015.7454565

[75] Xiao ZR, Lv BG, Wang X, Zhao YJ. A Healthcare Service System Based on Internet of Things. In: Proceeding of the Advanced Materials Research, 2013, pp. 1903-7. https://doi.org/10.4028/ www.scientific.net/AMR.774-776.1903

[76] Almehmadi T, Alshehri S, Tahir S. A Secure FogCloud Based Architecture for MIoT. In: Proceeding of the 2019 2nd International Conference on Computer Applications \& Information Security (ICCAIS), 2019, pp. 1-6. https://doi.org/10.1109/CAIS.2019.8769524

[77] Qi J, Yang P, Waraich A, Deng Z, Zhao Y, Yang Y. Examining sensor-based physical activity recognition and monitoring for healthcare using Internet of Things: A systematic review. Journal of Biomedical Informatics 2018; 87: 138-53. https://doi.org/10.1016/j.jbi.2018.09.002

[78] Bublitz FM, Oetomo A, Sahu KS, Kuang A, Fadrique LX, Velmovitsky PE, et al. Disruptive technologies for environment and health research: An overview of artificial intelligence, blockchain, and internet of things. International journal of environmental research and public health 2019; 16(20): 3847.

https://doi.org/10.3390/ijerph16203847

[79] Gupta BB, Quamara M. An overview of Internet of Things (IoT): Architectural aspects, challenges, and protocols. Concurr Comput. 2018.

https://doi.org/10.1002/cpe.4946

[80] Telecommunication standardization sector of ITU. Global information infrastructure, internet protocol aspects and next-generation networks next generation networks-frameworks and functional architecture models. Geneva, Switzerland: International Telecommunication Union, 2012. p. $1-14$.

[81] An BW, Shin JH, Kim S-Y, Kim J, Ji S, Park J, et al. Smart sensor systems for wearable electronic devices. Polymers. 2017; 9(8): 303. https://doi.org/10.3390/polym9080303

[82] Ni Q, Garcia Hernando AB, de la Cruz IP. The elderly's independent living in smart homes: A characterization of activities and sensing infrastructure survey to facilitate services development. Sensors (Basel, Switzerland) 2015; 15(5): 11312-62. https://doi.org/10.3390/s150511312

[83] Alarifi A, Al-Salman A, Alsaleh M, Alnafessah A, Al-Hadhrami S, Al-Ammar MA, et al. Ultra wideband indoor positioning technologies: Analysis and recent advances. Sensors (Basel, Switzerland) 2016; 16(5): 1-36.

https://doi.org/10.3390/s16050707

[84] Gholamhosseini L, Sadoughi F, Ahmadi H, Safaei A. Health Internet of Things: Strengths, Weakness, Opportunity, and Threats. In: Proceeding of the 2019 5th International Conference on Web Research (ICWR), Tehran, Iran, Iran 2019, pp. 287-96.

https://doi.org/10.1109/ICWR.2019.8765286

[85] Al-Sarawi S, Anbar M, Alieyan K, Alzubaidi M. Internet of Things (IoT) communication protocols: Review. In: Proceeding of the 2017 8th International Conference on Information Technology (ICIT), 2017, pp. 685-90. https://doi.org/10.1109/ICITECH.2017.8079928

[86] Chakkor S, Cheikh E, Mostafa B, Hajraoui A, Comparative Performance Analysis of Wireless Communication Protocols for Intelligent Sensors and Their Applications, International Journal of Advanced Computer Science and Applications. $2014 ; 5$.

https://doi.org/10.14569/IJACSA.2014.050413

[87] Verma A, Prakash S, Srivastava V, Kumar A, Mukhopadhyay SC, Sensing, Controlling, and IoT Infrastructure in Smart Building: A Review, IEEE Sensors J 2019; 19(20): 9036-46.

https://doi.org/10.1109/JSEN.2019.2922409

[88] Shakerighadi B, Anvari-Moghaddam A, Vasquez JC, Guerrero J. Internet of Things for Modern Energy Systems: State-of-the-Art, Challenges, and Open Issues. Energies 2018; 11.

https://doi.org/10.3390/en11051252

[89] Aqeel-ur-Rehman, Mehmood K, Baksh A. Communication Technology That Suits IoT - A Critical Review. In: Proceeding of the, Berlin, Heidelberg, 2013, pp. 14-25. https://doi.org/10.1007/978-3-642-41054-3_2

[90] Aly H, Elmogy M, Barakat S. Big Data on Internet of Things: Applications, Architecture, Technologies. Techniques, and Future Directions, Int J Comput Sci Eng 2015; 4: 300-13.

[91] Talavera JM, Tobón LE, Gómez JA, Culman MA, Aranda JM, Parra DT, et al., Review of IoT applications in agro-industrial and environmental fields, Computers and Electronics in Agriculture 2017; 142, Part A: 283-97.

https://doi.org/10.1016/j.compag.2017.09.015

[92] Gonzalez E, Pena R, Avila A, Vargas-Rosales C, Munoz-Rodriguez D. A Systematic Review on Recent Advances in mHealth Systems: Deployment Architecture for Emergency 
Response. Journal of healthcare engineering. 2017; 2017: 1-13. https://doi.org/10.1155/2017/9186270

[93] Khan R, Khan SU, Zaheer R, Khan S. Future internet: the internet of things architecture, possible applications and key challenges. In: Proceeding of the 2012 10th International Conference on Frontiers of Information Technology (FIT), 2012, pp. 257-60. https://doi.org/10.1109/FIT.2012.53

[94] Khoi NM, Saguna S, Mitra K, Ahlund C. IReHMo: An efficient IoT-based remote health monitoring system for smart regions. In: Proceeding of the 2015 17th International Conference on E-Health Networking, Application and Services, HealthCom. 2015, pp. 563-8. https://doi.org/10.1109/HealthCom.2015.7454565

[95] Datta SK, Bonnet C, Gyrard A, Costa RPFd, Boudaoud K. Applying Internet of Things for personalized healthcare in smart homes. In: Proceeding of the 2015 24th Wireless and Optical Communication Conference (WOCC), 2015, pp. 164-9. https://doi.org/10.1109/WOCC.2015.7346198

[96] Firdausi A. Overview the internet of things (IOT) system security, applications, architecture and business models. Indonesia: Universitas of Electrical Engineering; 2016.

[97] Darwish D. Improved Layered Architecture for Internet of Things. International Journal of Computing Academic Research (IJCAR). 2015; 4(4): 214-23.

[98] Mijac M, Androcec D, Picek R. Smart city services driven by IoT: A systematic review. Journal of Economic and Social Development. 2017; 4(2): 40-50.

[99] Tsai C-W, Lai C-F, Vasilakos AV. Future Internet of Things: open issues and challenges. Wireless Networks. 2014; 20(8): 2201-17. https://doi.org/10.1007/s11276-014-0731-0

[100] Erfannia L, Sadoughi F, Sheikhtaheri A. The advantages of implementing cloud computing in the health industry of Iran: A qualitative study. International Journal of Computer Science and Network Security. 2018; 18(1): 198-206.

[101] Thangaraj M, Ponmalar PP, Sujatha G, Anuradha $S$. Agent based semantic internet of things (IoT) in smart health care. In: Proceeding of the Proceedings of the The 11th International Knowledge Management in Organizations Conference on The changing face of Knowledge Management Impacting Society, Hagen, Germany, 2016 pp. 1-9. https://doi.org/10.1145/2925995.2926023

[102] Guo H, Ren J, Zhang D, Zhang Y, Hu J. A scalable and manageable IoT architecture based on transparent computing. Journal of Parallel and DistributedComputing 2018; 118: 5-13. https://doi.org/10.1016/j.jpdc.2017.07.003

[103] Dalli A, Bri S. Acquisition devices in internet of things: RFID and sensors, J Theor Appl Inf Technol 2016; 90(1): 194-200.
[104] Pacheco J, Zhu X, Badr Y, Hariri S. Enabling Risk Management for Smart Infrastructures with an Anomaly Behavior Analysis Intrusion Detection System. In: Proceeding of the 2017 IEEE 2nd International Workshops on Foundations and Applications of Self* Systems (FAS*W), 2017, pp. 324-8. https://doi.org/10.1109/FAS-W.2017.167

[105] Hossain MS, Muhammad G. Cloud-assisted Industrial Internet of Things (IIoT) - Enabled framework for health monitoring. Computer Networks 2016; 101: 192-202.

https://doi.org/10.1016/j.comnet.2016.01.009

[106] Bilal M, A Review of Internet of Things Architecture, Technologies and Analysis Smartphone-based Attacks Against 3D printers. arXiv preprint arXiv:170804560 2017.

[107] Vijayalakshmi A, Arockiam L, A study on security issues and challenges in IoT. Int J Eng Sci Manage Res. 2016;3(11):34-43.

[108] Ross R, Graubart R, Bodeau D, McQuaid R. Systems Security Engineering: Cyber Resiliency Considerations for the Engineering of Trustworthy Secure Systems. United States: NIST Special Publication 800-160, 2018 p. 1-142.

[109] Turkanovi'c M. Authentication and Key Agreement Protocol for Ad Hoc Networks Based on the Internet of Things Paradigm. 2016; 40 (1):153-4.

[110] Zhou X, Jin Z, Fu Y, Zhou H, Qin L. Short Signcryption Scheme for the Internet of Things. Informatica. 2011;35 (4): 521-30.

[111] Ebrahimi M, Sayadhaghighi M, Jolfaei A, Shamaeian N, Tadayon MH. A secure and decentralized trust management scheme for smart health systems. IEEE J Biomed Health Inform. Forthcoming 2021. https://doi.org/10.1109/JBHI.2021.3107339

[112] Alam T. IBchain: Internet of Things and Blockchain Integration Approach for Secure Communication in Smart Cities. 2021; 45(3):47786. https://doi.org/10.31449/inf.v45i3.3573 